\title{
Research Article \\ Equivariant Hopf Bifurcation in a Ring of Identical Cells with Delay
}

\section{Dejun Fan and Junjie Wei}

Department of Mathematics, Harbin Institute of Technology (Weihai), Weihai, Shandong 264209, China

Correspondence should be addressed to Junjie Wei, weijj@hit.edu.cn

Received 21 January 2009; Revised 27 March 2009; Accepted 24 May 2009

Recommended by Ji Huan He

\begin{abstract}
A kind of delay neural network with $n$ elements is considered. By analyzing the distribution of the eigenvalues, a bifurcation set is given in an appropriate parameter space. Then by using the theory of equivariant Hopf bifurcations of ordinary differential equations due to Golubitsky et al. (1988) and delay differential equations due to $\mathrm{Wu}$ (1998), and combining the normal form theory of functional differential equations due to Faria and Magalhaes (1995), the equivariant Hopf bifurcation is completely analyzed.
\end{abstract}

Copyright (c) 2009 D. Fan and J. Wei. This is an open access article distributed under the Creative Commons Attribution License, which permits unrestricted use, distribution, and reproduction in any medium, provided the original work is properly cited.

\section{Introduction}

The works of Golubitsky et al. [1, Chapter XVIII] have shown that rings of identical cells can lead to many interesting patterns of oscillation, which are predictable based on the theory of equivariant bifurcations. In a series of papers, Wu et al. [2-4] have extended the theory of equivariant Hopf bifurcation to delay differential equations. Recently, there has been interest in applying these results to neural networks, primarily to models related to the HopfieldCohen-Grossberg neural networks with time delays [5-9]. In a series of papers [10-23], the authors studied the Hopf bifurcation to a network with one or two delays. Particularly, Wu et al. [24] have investigated the synchronization and stable phase-locking in a delayed network with three identical neurons:

$$
\dot{x}_{i}(t)=-x_{i}(t)+\alpha f\left(x_{i}(t-\tau)\right)+\beta\left[f\left(x_{i-1}(t-\tau)\right)+f\left(x_{i+1}(t-\tau)\right)\right], \quad i(\bmod 3)
$$

where $f: R \rightarrow R$ is a sufficiently smooth sigmoid amplification function, normalized so that $f(0)=0$ and $f^{\prime}(0)=1, \alpha, \beta$, and $\tau$ are regarded as parameters. They have shown that in the region $A_{\mathrm{as}}=\{(\alpha, \beta):|\alpha-\beta|<1\}$ of the normalized parameters, system (4.1) is absolutely synchronous in the sense that every solution is convergent to the set of all 
synchronized phase states independent of the size of the time delay. The $\omega$-limit set of a given orbit can be either a synchronized equilibrium or a synchronized periodic solution, depending on the connection topology of the network, the strength of the self-connection and the neighborhood-interaction, and the size of the delay. On the other hand, they have shown that, in the region $\alpha-\beta<-1$, there exists a continuous surface $\tau=\tau(\alpha, \beta)$ where Hopf bifurcation of either a stable synchronized periodic solution or two stable phase-locked periodic solutions and six unstable periodic waves take place (more precisely, three mirrorreflecting waves and three standing waves). A question of mathematical and biological interest is whether the dynamics of (4.1) are possible for the following neural network model with $n$ elements:

$$
\dot{x}_{i}(t)=-x_{i}(t)+\alpha f\left(x_{i}(t-\tau)\right)+\beta\left[f\left(x_{i-1}(t-\tau)\right)+f\left(x_{i+1}(t-\tau)\right)\right], \quad i(\bmod n) .
$$

The purpose of the paper is to provide a detailed analysis of this question in the case that $n$ is an odd number. More precisely, we shall extend the main results of $\mathrm{Wu}$ et al. [24] to the system (1.2) with $n$ being an odd number. We are going to regard the delay $\tau$ as a parameter to investigate the dynamics of (1.2). According to the properties of the function $\tanh x$, we assume that the transfer function $f$ is adequately smooth, for example, $f \in C^{3}$, and satisfies the following normalization, monotonicity, concavity, and boundedness conditions:

(C1) $f(0)=0, f^{\prime}(0)=1$, and $f^{\prime}(x)>0$ for all $x \in R$;

(C2) $f^{\prime \prime}(0)=0$, and $x f^{\prime \prime}(x)<0$ for all $x \neq 0$;

(C3) $-\infty<\lim _{x \rightarrow \pm \infty} f(x)<\infty$;

(C4) $f^{\prime \prime \prime}(0)<0$.

At first, by analyzing the distribution of the eigenvalues, we give a bifurcation set in an appropriate parameter space to describe the stability of the equilibrium of the system (1.2) of how to change as the parameters change. Meanwhile, the equivariant Hopf bifurcations are found. Then, by employing the center manifold and normal form theory, the direction of the bifurcation and the stability of the bifurcating periodic solutions are determined.

The rest of this paper is organized as follows. In Section 2, the characteristic equation of the linearization of system (1.2) at the zero equilibrium is derived. In Section 3, by analyzing the distribution of the eigenvalues, a bifurcation set is given in the $(\alpha, \beta)$-plane. Then by using the theory of equivariant Hopf bifurcation to ordinary and delay differential equations due to Golubitsky et al. [1] and $\mathrm{Wu}$ [4], respectively, the local equivariant Hopf bifurcation of the trivial solution is completely analyzed. The appendix contain the detailed calculations of the normal forms on center manifolds of system (1.2) near Hopf bifurcation points.

We would like to mention that there are several articles on the bifurcation for $n$ dimensional neural network models with delays; we refer the reader to $[16,17,22]$ and references therein.

\section{The Characteristic Equations}

From conditions $(\mathrm{C} 1)-(\mathrm{C} 4)$, we know that the linearization of system (1.2) at an equilibrium $(0,0, \ldots, 0)^{T}$ is given by

$$
\dot{x}_{i}(t)=-x_{i}(t)+\alpha x_{i}(t-\tau)+\beta\left[x_{i-1}(t-\tau)+x_{i+1}(t-\tau)\right], \quad i(\bmod n) .
$$


The characteristic matrix is

$$
M_{n}(0, \lambda)=(\lambda+1) \operatorname{Id}-\alpha e^{-\lambda \tau} \mathrm{Id}-\beta e^{-\lambda \tau} \delta
$$

where Id is $n \times n$ identity matrix and

$$
\delta=\left(\begin{array}{ccccccc}
0 & 1 & 0 & 0 & \cdots & 0 & 1 \\
1 & 0 & 1 & 0 & \cdots & 0 & 0 \\
0 & 1 & 0 & 1 & \cdots & 0 & 0 \\
0 & 0 & 1 & 0 & \cdots & 0 & 0 \\
0 & 0 & 0 & 1 & \cdots & 0 & 0 \\
\vdots & \vdots & \vdots & \vdots & \cdots & \vdots & \vdots \\
1 & 0 & 0 & 0 & \cdots & 1 & 0
\end{array}\right)_{n \times n}
$$

Using a computation process similar to that of Wu et al. [24], Yuan and Campbell [25], we can obtain the following results.

Let

$$
x=e^{2 \pi i / n}, \quad v_{j}=\left(1, x^{j}, x^{2 j}, \ldots, x^{(n-1) j}\right)^{T} \quad(j=0,1, \ldots, n-1) .
$$

From

$$
x^{n j}=1, \quad x^{(n-1) j}=x^{-j}, \quad x^{(n-2) j}=x^{-2 j}, \ldots, \ldots
$$

it follows that

$$
\delta v_{j}=\left(\begin{array}{c}
x^{j}+x^{(n-1) j} \\
1+x^{2 j} \\
x^{j}+x^{3 j} \\
\vdots \quad \vdots \\
1+x^{(n-2) j}
\end{array}\right)=\left(x^{j}+x^{-j}\right) v_{j}=2 \cos \left(\frac{2 \pi j}{n}\right) v_{j}
$$

Hence,

$$
\begin{aligned}
M_{n}(0, \lambda) v_{j} & =\left[\lambda+1-\left(\alpha+\beta\left(x^{j}+x^{-j}\right)\right) e^{-\lambda \tau}\right] v_{j} \\
& =\left[\lambda+1-\left(\alpha+2 \beta \cos \frac{2 \pi j}{n}\right) e^{-\lambda \tau}\right] v_{j}
\end{aligned}
$$


and the characteristic equation is

$$
\begin{aligned}
\operatorname{det} M_{n}(0, \lambda) & =\prod_{j=0}^{n-1}\left[\lambda+1-\left(\alpha+2 \beta \cos \frac{2 \pi j}{n}\right) e^{-\lambda \tau}\right] \\
& =\Delta_{0}(\lambda) \times \prod_{j=1}^{n-1} \Delta_{j}(\lambda) \\
& =0
\end{aligned}
$$

where

$$
\Delta_{j}(\lambda)=\lambda+1-\left(\alpha+2 \beta \cos \frac{2 \pi j}{n}\right) e^{-\lambda \tau}, \quad(j=0,1,2, \ldots, n-1) .
$$

We observe that (2.7) can be simplified, further elucidating the structure of characteristic equation.

(I) When $n$ is an odd number, we obtain

$$
\begin{aligned}
\operatorname{det} M_{n}(0, \lambda) & =\Delta_{0}(\lambda) \times \prod_{j=1}^{(n-1) / 2} \Delta_{j}^{2}(\lambda) \\
& =\left[\lambda+1-(\alpha+2 \beta) e^{-\lambda \tau}\right] \times \prod_{j=1}^{(n-1) / 2}\left[\lambda+1-\left(\alpha+2 \beta \cos \frac{2 \pi j}{n}\right) e^{-\lambda \tau}\right]^{2} .
\end{aligned}
$$

(II) When $n$ is an even number, we obtain

$$
\begin{aligned}
\operatorname{det} M_{n}(0, \lambda)= & \Delta_{0}(\lambda) \Delta_{n / 2}(\lambda) \times \prod_{j=1}^{(n-2) / 2} \Delta_{j}^{2}(\lambda) \\
= & {\left[\lambda+1-(\alpha+2 \beta) e^{-\lambda \tau}\right]\left[\lambda+1-(\alpha-2 \beta) e^{-\lambda \tau}\right] } \\
& \times \prod_{j=1}^{(n-2) / 2}\left[\lambda+1-\left(\alpha+2 \beta \cos \frac{2 \pi j}{n}\right) e^{-\lambda \tau}\right]^{2} .
\end{aligned}
$$

In the case when $n$ is even, that is, $n=2 m$, $\operatorname{det} M_{n}(0, \lambda)$ may be further simplified into two subcases. 
(i) When $m$ is an odd number, we have

$$
\begin{aligned}
\operatorname{det} M_{n}(0, \lambda)= & \Delta_{0}(\lambda) \Delta_{n / 2}(\lambda) \times \prod_{j=1}^{(m-1) / 2} \Delta_{j}^{2}(\lambda) \Delta_{m-j}^{2}(\lambda) \\
= & {\left[\lambda+1-(\alpha+2 \beta) e^{-\lambda \tau}\right]\left[\lambda+1-(\alpha-2 \beta) e^{-\lambda \tau}\right] } \\
& \times \prod_{j=1}^{(m-1) / 2}\left[\lambda+1-\left(\alpha+2 \beta \cos \frac{\pi j}{m}\right) e^{-\lambda \tau}\right]^{2}\left[\lambda+1-\left(\alpha-2 \beta \cos \frac{\pi j}{m}\right) e^{-\lambda \tau}\right]^{2} .
\end{aligned}
$$

(ii) When $m$ is an even number, we have

$$
\begin{aligned}
\operatorname{det} M_{n}(0, \lambda)= & \Delta_{0}(\lambda) \Delta_{n / 2}(\lambda) \Delta_{n / 4}^{2}(\lambda) \times \prod_{j=1}^{(m-2) / 2} \Delta_{j}^{2}(\lambda) \Delta_{m-j}^{2}(\lambda) \\
= & {\left[\lambda+1-(\alpha+2 \beta) e^{-\lambda \tau}\right]\left[\lambda+1-(\alpha-2 \beta) e^{-\lambda \tau}\right]\left(\lambda+1-\alpha e^{-\lambda \tau}\right)^{2} } \\
& \times \prod_{j=1}^{(m-2) / 2}\left[\lambda+1-\left(\alpha+2 \beta \cos \frac{\pi j}{m}\right) e^{-\lambda \tau}\right]^{2}\left[\lambda+1-\left(\alpha-2 \beta \cos \frac{\pi j}{m}\right) e^{-\lambda \tau}\right]^{2} .
\end{aligned}
$$

From (2.8)-(2.13), we obtain the characteristic equation of (1.2) given by the following.

(1) When $n$ is an odd number,

$$
\left[\lambda+1-(\alpha+2 \beta) e^{-\lambda \tau}\right] \prod_{j=1}^{(n-1) / 2}\left[\lambda+1-\left(\alpha+2 \beta \cos \frac{2 \pi j}{n}\right) e^{-\lambda \tau}\right]^{2}=0
$$

(2) When $n$ is an even number and $n$ is not the multiple of 4 ,

$$
\begin{aligned}
{[\lambda+1} & \left.-(\alpha+2 \beta) e^{-\lambda \tau}\right] \prod_{j=1}^{(n-2) / 4}\left[\lambda+1-\left(\alpha+2 \beta \cos \frac{2 \pi j}{n}\right) e^{-\lambda \tau}\right]^{2} \\
& \times\left[\lambda+1-(\alpha-2 \beta) e^{-\lambda \tau}\right] \prod_{j=1}^{(n-2) / 4}\left[\lambda+1-\left(\alpha-2 \beta \cos \frac{2 \pi j}{n}\right) e^{-\lambda \tau}\right]^{2} \\
= & 0 .
\end{aligned}
$$


(3) When $n$ is the multiple of 4 ,

$$
\begin{aligned}
{[\lambda+1} & \left.-(\alpha+2 \beta) e^{-\lambda \tau}\right] \prod_{j=1}^{(n-4) / 4}\left[\lambda+1-\left(\alpha+2 \beta \cos \frac{2 \pi j}{n}\right) e^{-\lambda \tau}\right]^{2} \\
& \times\left[\lambda+1-(\alpha-2 \beta) e^{-\lambda \tau}\right] \prod_{j=1}^{(n-4) / 4}\left[\lambda+1-\left(\alpha-2 \beta \cos \frac{2 \pi j}{n}\right) e^{-\lambda \tau}\right]^{2} \times\left(\lambda+1-\alpha e^{-\lambda \tau}\right)^{2} \\
= & 0 .
\end{aligned}
$$

\section{Bifurcation Analysis with $n$ being an Odd Number}

In this section, we are going to analyze the distribution of the root of the characteristic equations given in previous section, respectively.

In this case, the characteristic equation of (1.2) is

$$
\left[\lambda+1-(\alpha+2 \beta) e^{-\lambda \tau}\right] \prod_{j=1}^{(n-1) / 2}\left[\lambda+1-\left(\alpha+2 \beta \cos \frac{2 \pi j}{n}\right) e^{-\lambda \tau}\right]^{2}=0 .
$$

Clearly, $i \omega(\omega>0)$ is a root of (3.1) if and only if there exists a

$$
j \in\left\{0,1,2, \ldots, \frac{n-1}{2}\right\}
$$

such that $\omega$ satisfies

$$
i \omega+1-\left(\alpha+2 \beta \cos \frac{2 \pi j}{n}\right) e^{-i \omega \tau}=0,
$$

that is,

$$
\cos \omega \tau=\frac{1}{\alpha+2 \beta \cos (2 \pi j / n)}, \quad \sin \omega \tau=-\frac{\omega}{\alpha+2 \beta \cos (2 \pi j / n)} .
$$

This leads to

$$
\omega_{j}=\sqrt{\left(\alpha+2 \beta \cos \frac{2 \pi j}{n}\right)^{2}-1}
$$

This shows that the necessary condition for (3.1) possessing purely imaginary roots is that there exists $j \in\{0,1,2, \ldots,(n-1) / 2\}$ such that $|\alpha+2 \beta \cos (2 \pi j / n)|>1$, that is,

$$
\alpha+2 \beta \cos \frac{2 \pi j}{n}>1 \quad \text { or } \quad \alpha+2 \beta \cos \frac{2 \pi j}{n}<-1
$$

From (3.1), conclusion then follows immediately. 
Proposition 3.1. Equation (3.1) has at most $(n+1) / 2$ pairs of purely imaginary roots when $n$ is an odd number.

Without loss of generality, one assumes the (3.1) has $(n+1) / 2$ pairs of purely imaginary roots $\pm \omega_{j}(j=0,1,2, \ldots,(n-1) / 2)$ exactly. Define

$$
\tau_{k}^{(j)}=\frac{1}{\omega_{j}}\left[\arccos \frac{1}{\alpha+2 \beta \cos (2 \pi j / n)}+2 k \pi\right], \quad j=0,1, \ldots, \frac{n-1}{2} ; k=0,1,2, \ldots
$$

Then $\pm i \omega_{j}$ are purely imaginary roots of (3.1) with $\tau=\tau_{k}^{(j)}(k=0,1,2, \ldots)$, respectively. one notes that $\omega_{j} \tau_{k}^{(j)} \in(0, \pi]($ resp., $\in(\pi, 2 \pi])$ when

$$
\sin \left(\omega_{j} \tau_{k}^{(j)}\right)=-\frac{\omega_{j}}{\alpha+2 \beta \cos (2 \pi j / n)}>0 \quad(\text { resp. }<0)
$$

We can obtain the following result with no difficulty.

Proposition 3.2. Let $\lambda(\tau)=\sigma(\tau) \pm i \omega(\tau)$ be the root of (3.1) near $\tau=\tau_{k}^{(j)}$ satisfying $\sigma\left(\tau_{k}^{(j)}\right)=0$, $\omega\left(\tau_{k}^{(j)}\right)=\omega_{j}(j=0,1, \ldots,(n-1) / 2 ; k=0,1,2, \ldots)$. Then

$$
\left.\frac{d \sigma(\tau)}{d \tau}\right|_{\tau=\tau_{k}^{(j)}}>0
$$

Proof. From (3.1) we have

$$
\lambda+1-\left(\alpha+2 \beta \cos \frac{2 \pi j}{n}\right) e^{-\lambda \tau}=0
$$

Differentiating both sides with respect to $\tau$, we have

$$
\left(\frac{d \lambda}{d \tau}\right)^{-1}=-\frac{e^{\lambda \tau}}{\lambda(\alpha+2 \beta \cos (2 \pi j / n))}-\frac{\tau}{\lambda}
$$

Notice that $\lambda\left(\tau_{k}^{(j)}\right)=i \omega_{j}$, it follows that

$$
\left.\operatorname{Re} \frac{d \lambda}{d \tau}\right|_{\tau=\tau_{k}^{(j)}}=\left.\frac{d \sigma(\tau)}{d \tau}\right|_{\tau=\tau_{k}^{(j)}}=\frac{\omega_{j}^{2}}{\left(1+\tau_{k}^{(j)}\right)^{2}+\left(\tau_{k}^{(j)} \omega_{j}\right)^{2}}>0
$$

This completes the proof. 
When $\tau=0$, (3.1) becomes

$$
[\lambda+1-(\alpha+2 \beta)] \prod_{j=1}^{(n-1) / 2}\left[\lambda+1-\left(\alpha+2 \beta \cos \frac{2 \pi j}{n}\right)\right]^{2}=0
$$

Its roots are given by

$$
\begin{aligned}
\lambda_{0} & =\alpha+2 \beta-1 \\
\lambda_{1} & =\alpha+2 \beta \cos \frac{2 \pi}{n}-1, \\
\lambda_{2} & =\alpha+2 \beta \cos \frac{4 \pi}{n}-1, \\
\vdots & \vdots \quad \vdots \\
\lambda_{(n-1) / 2} & =\alpha+2 \beta \cos \frac{(n-1) \pi}{n}-1 \\
& =\alpha-2 \beta \cos \frac{\pi}{n}-1 .
\end{aligned}
$$

For convenience, denote

$$
\begin{gathered}
S:=\left\{(\alpha, \beta) \mid \alpha<1, \frac{1-\alpha}{2 \cos ((n-1) \pi / n)}<\beta<\frac{1-\alpha}{2}\right\}, \\
P:=\left\{(\alpha, \beta) \mid \frac{-1-\alpha}{2}<\beta<\frac{1-\alpha}{2}, \frac{1-\alpha}{2 \cos ((n-1) \pi / n)}<\beta<\frac{-1-\alpha}{2 \cos ((n-1) \pi / n)}\right\},
\end{gathered}
$$

and let $D:=S / P$. One can find out that the region $S$ is a sector, $P$ is a parallelogram, and $D$ is the outside of the parallelogram in the sector $S$. These are shown in Figure 1.

Proposition 3.3. If $\alpha+2 \beta<1$ and $\alpha+2 \beta \cos ((n-1) \pi / n)<1(\alpha-2 \beta \cos (\pi / n)<1)$, that is $(\alpha, \beta) \in S$, then all roots of (3.13) have negative real parts.

From (3.6), Proposition 3, and Corollary 2.4 of Ruan and Wei [19], we have the following result immediately.

Proposition 3.4. If $|\alpha+2 \beta|<1$ and $|\alpha+2 \beta \cos ((n-1) \pi / n)|<1(|\alpha-2 \beta \cos (\pi / n)|<1)$, that is, $(\alpha, \beta) \in P$, then all roots of (3.1) have negative real parts for all $\tau \geq 0$.

From Proposition 2 and Corollary 2.4 of Ruan and Wei [19], conclusion then follows immediately.

Proposition 3.5. If there exist $j \in\{0,1,2, \ldots,(n-1) / 2\}$ such that $\alpha+2 \beta \cos (2 \pi j / n)>1$, that is, $(\alpha, \beta)$ is located outside of the sector $S$, then (3.1) has at least one root with positive part for all $\tau \geq 0$. 


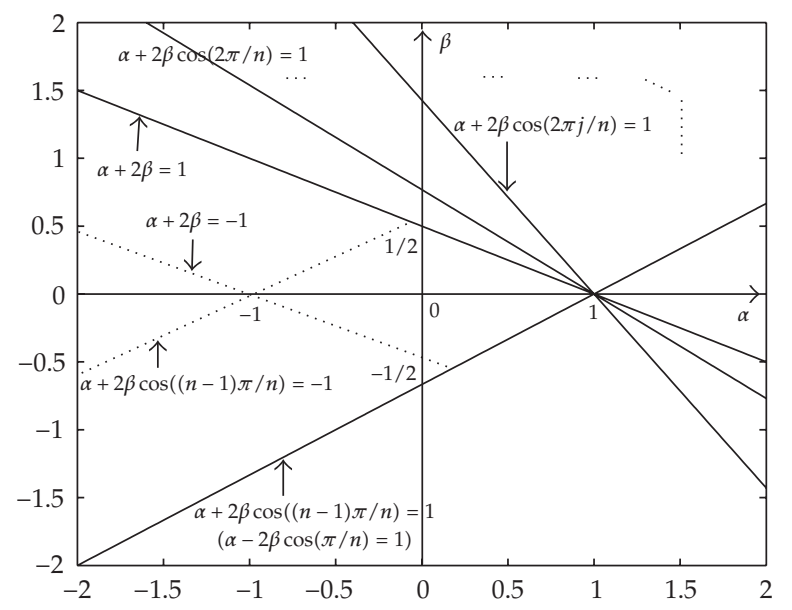

Figure 1: Bifurcation set in $(\alpha, \beta)$-plane. The outside of the sector $S$ is unstable region; the parallelogram $P$ is the absolutely stable region; the outside of the parallelogram in the sector is conditionally stable region.

Proposition 3.6. Suppose that one of the following conditions is satisfied (i.e., $(\alpha, \beta) \in D$ ):

(1) $|\alpha+2 \beta|<1$ and $\alpha-2 \beta \cos (\pi / n)<-1$;

(2) $\alpha+2 \beta<-1$ and $|\alpha-2 \beta \cos (\pi / n)|<1$;

(3) $\alpha+2 \beta<-1$ and $\alpha-2 \beta \cos (\pi / n)<-1$.

Then there exist at most $(n+1) / 2$ sequences of critical value

$$
\tau_{k}^{(j)}, \quad j=0,1, \ldots, \frac{n-1}{2} ; k=0,1,2, \ldots
$$

defined by (3.7) such that

(1) all roots of (3.1) have negative real parts for all $\tau \in\left[0, \tau_{0}\right)$, where

$$
\tau_{0}=\min \left\{\tau_{0}^{(j)}: 0 \leq j \leq \frac{n-1}{2}\right\}
$$

(2) equation (3.1) has at least one root with positive part when $\tau>\tau_{0}$;

(3) at $\tau=\tau_{k}^{(j)}(j=0,1, \ldots,(n-1) / 2 ; k=0,1, \ldots),(3.1)$ has a pair of purely imaginary roots $\pm i \omega_{j}$.

From Propositions 3.1-3.6, we have the following results immediately.

Theorem 3.7. (i) If $|\alpha+2 \beta|<1$ and $|\alpha-2 \beta \cos (\pi / n)|<1$, then the zero equilibrium of (1.2) is asymptotically stable for all $\tau \geq 0$.

(ii) If $j(j=0,1,2, \ldots,(n-1) / 2)$ exist such that $\alpha+2 \beta \cos (2 \pi j / n)>1$, then the zero equilibrium of (1.2) is unstable for all $\tau \geq 0$. 
(iii) Suppose that one of the following conditions holds:

(1) $|\alpha+2 \beta|<1$ and $\alpha-2 \beta \cos (\pi / n)<-1$;

(2) $\alpha+2 \beta<-1$ and $|\alpha-2 \beta \cos (\pi / n)|<1$;

(3) $\alpha+2 \beta<-1$ and $\alpha-2 \beta \cos (\pi / n)<-1$.

Then there exist at most $(n+1) / 2$ sequences of critical value $\tau_{k}^{(j)}(j=0,1, \ldots,(n-1) / 2 ; k=$ $0,1,2, \ldots)$ defined by (3.7) such that the zero equilibrium of (1.2) is asymptotically stable for $\tau \in$ $\left[0, \tau_{0}\right)$, where $\tau_{0}=\min \left\{\tau_{0}^{(j)}: 0 \leq j \leq(n-1) / 2\right\}$, unstable for all $\tau>\tau_{0}$, and the system undergoes $a$ Hopf bifurcation with $\tau=\tau_{k}^{(j)}(j=0,1, \ldots,(n-1) / 2 ; k=0,1, \ldots)$.

Proposition 3.6 shows that the region $D$, which is the outside of the parallelogram in the sector shown as in Figure 1, is a conditionally stable region. This means that the stability of the zero equilibrium of (1.2) is dependent on the delay. Namely, the distribution of the roots of (3.1) is dependent on the delay. Particularly, for $(\alpha, \beta) \in D$, (3.1) with some critical value $\tau$ has simple pure imaginary roots or multiply pure imaginary roots. It is well know that the Hopf bifurcations are different between the simple and multiple pure imaginary roots. So it is necessary to observe the region $D$. In fact, we have the following conclusions on occurrence of purely imaginary roots of (3.1) for $(\alpha, \beta) \in D$.

(1) In the region

$$
E_{0}=\left\{(\alpha, \beta) \mid \alpha+2 \beta<-1 ; \alpha-2 \beta \cos \frac{\pi}{n}<1 ; \alpha+2 \beta \cos \frac{2 \pi}{n}>-1\right\}
$$

Equation (3.1) with $\tau_{k}^{(0)}$ has one pair of purely imaginary roots exactly, denoted by $\pm i \omega_{0}$, which are simple, where $\omega_{0}=\sqrt{(\alpha+2 \beta)^{2}-1}$ and

$$
\tau_{k}^{(0)}=\frac{1}{\omega_{0}}\left(\arccos \frac{1}{\alpha+2 \beta}+2 k \pi\right) \quad(k=0,1,2, \ldots) .
$$

(2) In the region

$$
E_{1}=\left\{(\alpha, \beta) \mid \alpha+2 \beta \cos \frac{2 \pi}{n}<-1 ; \alpha-2 \beta \cos \frac{\pi}{n}<1 ; \alpha+2 \beta \cos \frac{4 \pi}{n}>-1\right\},
$$

Equation (3.1) has two pairs of purely imaginary roots exactly, denoted by $\pm i \omega_{0}$ and $\pm i \omega_{1}$, when $\tau=\tau_{k}^{(1)}$ and $\tau=\tau_{k}^{(0)}$, respectively. Here $\pm i \omega_{0}$ are simple, $\pm i \omega_{1}$ are double, and $\omega_{1}=$ $\sqrt{(\alpha+2 \beta \cos (2 \pi / n))^{2}-1}, \tau_{k}^{(0)}$ is defined by (3.20) and

$$
\tau_{k}^{(1)}=\frac{1}{\omega_{1}}\left(\arccos \frac{1}{\alpha+2 \beta \cos (2 \pi / n)}+2 k \pi\right) \quad(k=0,1,2, \ldots) .
$$

Furthermore,

$$
\tau_{0}^{(0)}=\frac{1}{\omega_{0}}\left(\arccos \frac{1}{\alpha+2 \beta}\right)>\tau_{0}^{(1)}=\frac{1}{\omega_{1}}\left(\arccos \frac{1}{\alpha+2 \beta \cos (2 \pi / n)}\right) .
$$


(3) In the region

$$
E_{2}=\left\{(\alpha, \beta) \mid \alpha+2 \beta \cos \frac{4 \pi}{n}<-1 ; \alpha-2 \beta \cos \frac{\pi}{n}<1 ; \alpha+2 \beta \cos \frac{6 \pi}{n}>-1\right\},
$$

Equation (3.1) has three pairs of purely imaginary roots exactly, denoted by $\pm i \omega_{0}, \pm i \omega_{1}$, and $\pm i \omega_{2}$, when $\tau=\tau_{k}^{(0)}, \tau=\tau_{k}^{(1)}$, and $\tau=\tau_{k}^{(2)}$, respectively. Here $\pm i \omega_{0}$ are simple, $\pm i \omega_{1}$ and $\pm i \omega_{2}$ are double, $\omega_{2}=\sqrt{(\alpha+2 \beta \cos (4 \pi / n))^{2}-1}, \tau_{k}^{(0)}$ and $\tau_{k}^{(1)}$ are defined by (3.20) and (3.22), respectively, and

$$
\tau_{k}^{(2)}=\frac{1}{\omega_{2}}\left(\arccos \frac{1}{\alpha+2 \beta \cos (4 \pi / n)}+2 k \pi\right) \quad(k=0,1,2, \ldots) .
$$

Furthermore,

$$
\tau_{0}^{(0)}>\tau_{0}^{(1)}>\tau_{0}^{(2)}=\frac{1}{\omega_{2}}\left(\arccos \frac{1}{\alpha+2 \beta \cos (4 \pi / n)}\right) .
$$

Naturally, the region $E_{(n-1) / 4}$ (when $n-1$ is multiple of 4 ) or $E_{(n-3) / 4}$ (when $n-1$ is not multiple of 4) can be defined by

$$
\begin{aligned}
& E_{(n-1) / 4}=\left\{(\alpha, \beta) \mid \alpha+2 \beta \cos \frac{(n-1) \pi}{2 n}<-1 ; \alpha-2 \beta \cos \frac{\pi}{n}<1 ; \alpha+2 \beta \cos \frac{2 \pi}{n}<-1\right\}, \\
& E_{(n-3) / 4}=\left\{(\alpha, \beta) \mid \alpha+2 \beta \cos \frac{(n-3) \pi}{2 n}<-1 ; \alpha-2 \beta \cos \frac{\pi}{n}<1 ; \alpha+2 \beta \cos \frac{2 \pi}{n}<-1\right\} .
\end{aligned}
$$

$\star A$ : Obviously, in the region $E_{(n-1) / 4}\left(\right.$ or $\left.E_{(n-3) / 4}\right)$, we have that

$$
\tau_{0}^{(0)}>\tau_{0}^{(1)}>\tau_{0}^{(2)}>\tau_{0}^{(3)}>\cdots>\tau_{0}^{((n-1) / 4)} \quad\left(\text { or } \tau_{0}^{((n-3) / 4)}\right) .
$$

(4) In the region

$$
\begin{aligned}
E_{((n-1) / 2)} & =\left\{(\alpha, \beta) \mid \alpha+2 \beta \cos \frac{(n-1) \pi}{n}<-1 ; \alpha+2 \beta<1 ; \alpha+2 \beta \cos \frac{(n-3) \pi}{n}>-1\right\} \\
& =\left\{(\alpha, \beta) \mid \alpha-2 \beta \cos \frac{\pi}{n}<-1 ; \alpha+2 \beta<1 ; \alpha-2 \beta \cos \frac{3 \pi}{n}>-1\right\},
\end{aligned}
$$

Equation (3.1) has a pair of purely imaginary roots exactly, denoted by $\pm i \omega_{(n-1) / 2}$, which are double, where $\omega_{(n-1) / 2}=\sqrt{(\alpha+2 \beta \cos ((n-1) \pi / n))^{2}-1}=\sqrt{(\alpha-2 \beta \cos (\pi / n))^{2}-1}$ and

$$
\tau_{k}^{((n-1) / 2)}=\frac{1}{\omega_{(n-1) / 2}}\left(\arccos \frac{1}{\alpha-2 \beta \cos (\pi / n)}+2 k \pi\right) \quad(k=0,1,2, \ldots) .
$$


(5) In the region

$$
E_{((n-3) / 2)}=\left\{(\alpha, \beta) \mid \alpha-2 \beta \cos \frac{3 \pi}{n}<-1 ; \alpha+2 \beta<1 ; \alpha-2 \beta \cos \frac{5 \pi}{n}>-1\right\},
$$

Equation (3.1) has two pairs of purely imaginary roots exactly, denoted by $\pm i \omega_{(n-1) / 2}$ and $\pm i \omega_{(n-3) / 2}$, when $\tau=\tau_{k}^{((n-1) / 2)}$ and $\tau=\tau_{k}^{((n-3) / 2))}$, respectively, and the two pairs of purely imaginary roots are all double. Here

$$
\omega_{(n-3) / 2}=\sqrt{\left(\alpha+2 \beta \cos \frac{(n-3) \pi}{n}\right)^{2}-1}=\sqrt{\left(\alpha-2 \beta \cos \frac{3 \pi}{n}\right)^{2}-1},
$$

$\tau_{k}^{((n-1) / 2)}$ is defined by (3.30), and

$$
\tau_{k}^{((n-3) / 2)}=\frac{1}{\omega_{(n-3) / 2}}\left(\arccos \frac{1}{\alpha-2 \beta \cos (3 \pi / n)}+2 k \pi\right) \quad(k=0,1,2, \ldots) .
$$

Moreover,

$$
\tau_{0}^{((n-1) / 2)}=\frac{1}{\omega_{(n-1) / 2}}\left(\arccos \frac{1}{\alpha-2 \beta \cos (\pi / n)}\right)>\tau_{0}^{((n-3) / 2)}=\frac{1}{\omega_{(n-3) / 2}}\left(\arccos \frac{1}{\alpha-2 \beta \cos (3 \pi / n)}\right) .
$$

Naturally, the region $E_{(n+3) / 4}$ (when $n-1$ is multiple of 4 ) or $E_{(n+1) / 4}$ (when $n-1$ is not multiple of 4) can be defined by

$$
\begin{aligned}
& E_{(n+3) / 4}=\left\{(\alpha, \beta) \mid \alpha+2 \beta \cos \frac{2 \pi}{n}<-1 ; \alpha+2 \beta<1 ; \alpha+2 \beta>-1\right\}, \\
& E_{(n+1) / 4}=\left\{(\alpha, \beta) \mid \alpha+2 \beta \cos \frac{2 \pi}{n}<-1 ; \alpha+2 \beta<1 ; \alpha+2 \beta>-1\right\} .
\end{aligned}
$$

$\star B$ : Obviously, in the region $E_{(n+3) / 4}\left(\right.$ or $\left.E_{(n+1) / 4}\right)$, we have

$$
\tau_{0}^{((n-1) / 2)}>\tau_{0}^{((n-3) / 2)}>\tau_{0}^{((n-5) / 2)}>\cdots>\tau_{0}^{((n+3) / 4)} \quad\left(\text { or } \tau_{0}^{((n+1) / 4)}\right) .
$$

To carry out our work we need some background from the theory of functional differential equation. Let $C=C\left([-\tau, 0], R^{n}\right)$ denote the Banach space of continuous mappings from $[-\tau, 0]$ into $R^{n}$ equipped with the supernorm. Let $x(t)$ be a solution of (1.2) and define $x_{t}(\theta)=x(t+\theta),-\tau \leq \theta \leq 0$. If $x(t)$ is continuous, then $x_{t}(\theta) \in C$. With this structure, we may write the model as the following functional differential equation:

$$
\dot{x}(t)=F\left(x_{t}\right),
$$


where $F: C \rightarrow R^{n}$ is defined via

$$
F_{i}(\phi)=-\phi_{i}(0)+\alpha f\left(\phi_{i}(-\tau)\right)+\beta\left[f\left(\phi_{i-1}(-\tau)\right)+f\left(\phi_{i+1}(-\tau)\right)\right], \quad i(\bmod n) .
$$

Similarly, the linearization of $(3.37)$ at equilibrium $(0,0, \ldots, 0)^{T}$ may be written as

$$
\dot{u}(t)=L(\tau) u_{t}
$$

where the linear operator $L(\tau): C \rightarrow R^{n}$ is defined via

$$
L(\tau) \phi=-\operatorname{Id} \phi(0)+\alpha \operatorname{Id} \phi(-\tau)+\beta \delta \phi(-\tau) .
$$

It is well known that a linear functional differential equation such as (3.39) generates a strongly continuous semigroup of linear operators with infinitesimal generator $A$ given by

$$
A \phi=\dot{\phi}, \quad \phi \in \operatorname{Dom}(A),
$$

where $\operatorname{Dom}(A)=\phi \in C, \phi(0)=L(\tau) \phi$.

To explore the possible (spatial) symmetry of (1.2), we need to introduce three compact Lie groups. One is the cycle group $S^{1}$; another is $Z_{n}$, the cyclic group of order $n$, which corresponds to rotations of $2 \pi / n$, denoting the generator of this group by $\rho$, then action on $R^{n}$ is given by $(\rho x)_{i}=x_{i+1}$; the third is the dihedral group $D_{n}$ of order $2 n$, which corresponds to the group of symmetries of an $n$-gon. It can be shown that $D_{n}$ is generated by $\rho$ and $\mathcal{\kappa}$, where $\mathcal{\kappa}$ is the flip of order 2 or reflection, and it acts on $R^{n}$ by $(\kappa x)_{i}=x_{n+2-i}$.

Definition 3.8. Let $F: C \rightarrow R^{n}$ and $\Gamma$ be a compact group. The system (3.37) is said to be $\Gamma$-equivariant if $F\left(\gamma x_{t}\right)=\gamma F\left(x_{t}\right)$ for all $\gamma \in \Gamma$.

Proposition 3.9. The nonlinear system (3.37) and linear system (3.39) are $D_{n}$ equivariant.

Proof. We begin with (3.37); that is, we let $F$ be as in (3.38) and $\phi \in C$. We need only check the equivariant condition on the generators, $\rho, \kappa$, of $D_{n}$ :

$$
\begin{aligned}
F_{i}(\rho \phi) & =-(\rho \phi)_{i}(0)+\alpha f\left((\rho \phi)_{i}(-\tau)\right)+\beta\left[f\left((\rho \phi)_{i-1}(-\tau)\right)+f\left((\rho \phi)_{i+1}(-\tau)\right)\right] \\
& =-\phi_{i+1}(0)+\alpha f\left(\phi_{i+1}(-\tau)\right)+\beta\left[f\left(\phi_{i}(-\tau)\right)+f\left(\phi_{i+2}(-\tau)\right)\right] \\
& =F_{i+1}(\phi) \\
& =\rho F_{i}(\phi), \\
F_{i}(\kappa \phi) & =-(\kappa \phi)_{i}(0)+\alpha f\left((\kappa \phi)_{i}(-\tau)\right)+\beta\left[f\left((\kappa \phi)_{i-1}(-\tau)\right)+f\left((\kappa \phi)_{i+1}(-\tau)\right)\right] \\
& =-\phi_{n+2-i}(0)+\alpha f\left(\phi_{n+2-i}(-\tau)\right)+\beta\left[f\left(\phi_{n+3-i}(-\tau)\right)+f\left(\phi_{n+1-i}(-\tau)\right)\right] \\
& =F_{n+2-i}(\phi) \\
& =\kappa F_{i}(\phi) .
\end{aligned}
$$

Thus (3.37) is $D_{n}$ equivariant. 
From (3.39), we begin by noting that $L$ may be written componentwise as follows:

$$
L_{i}(\tau) \phi=-\phi_{i}(0)+a \phi_{i}(-\tau)+\beta\left[\phi_{i-1}(-\tau)+\phi_{i+1}(-\tau)\right], \quad i(\bmod n) .
$$

The rest of the proof is similar to that for (3.37). This completes the proof.

In the case where $(\alpha, \beta) \in E_{0}$, we can apply the standard Hopf bifurcation theorem of delay differential equations to obtain a Hopf bifurcation of synchronous periodic solutions. In the case where $(\alpha, \beta) \in E_{j}(1 \leq j \leq(n-1) / 2)$, the aforementioned standard Hopf bifurcation theorem does not apply since $\pm i \omega_{j}$ are double eigenvalues. On the other hand, the considered system is equivariant with respect to the $D_{n}$-action where the $Z_{n}$ subgroup acts by permutation (sending $x_{i}$ to $x_{i+1}$ ) and the flip acts by interchanging (sending $x_{i}$ to $x_{n+2-i}$ ). This allows us to apply the symmetric Hopf bifurcation theorem for delay differential equations established in [4] (as an extension of the well-known Golubitsky-Stewart Theorem [1] for symmetric ordinary differential equations) to obtain $2(n+1)$ branches of asynchronous periodic solutions. More precisely, we have the following theorem.

Theorem 3.10. Assume that $(\alpha, \beta) \in D(D=S / P$, where the regions $S$ and $P$ are defined by (3.15) and (3.16), resp.). Then

(1) in case $(\alpha, \beta) \in E_{0}$, near $\tau=\tau_{0}^{(0)}$ there exists a supercritical bifurcation of stable synchronous periodic solutions of period $p_{\tau}$ near $\left(2 \pi / \omega_{0}\right)$, bifurcated from the zero solution of system (1.2);

(2) in case $(\alpha, \beta) \in E_{j}(1 \leq j \leq(n-1) / 2)$, near $\tau=\tau_{0}^{(j)}$ there exist $2(n+1)$ branches of asynchronous periodic solutions of period $p_{\tau}$ near $\left(2 \pi / \omega_{j}\right)$, bifurcated simultaneously from the zero solution of system (1.2), and there are

(a) two stable phase-locked oscillations: $x_{i}(t)=x_{i+1}\left(t \pm\left(p_{\tau / n}\right)\right)$, for $i(\bmod n)$ and $t \in R$,

(b) $n$ unstable mirror-reflecting waves: $x_{i}(t)=x_{n+2 k-i}(t)$ for $i(\bmod n)$ and $t \in R$, where $k=1,2, \ldots, n$,

(c) $n$ unstable standing waves: $x_{i}(t)=x_{n+2 k-i}\left(t-\left(p_{\tau} / 2\right)\right)$ for $i(\bmod n)$ and $t \in R$, where $k=1,2, \ldots, n$.

Proof. (1) The existence is an immediate application of the standard Hopf bifurcation theorem for functional differential equations. Let $\tau^{*}=\tau_{0}^{(0)}, \mu=\tau-\tau_{0}^{(0)}$. According to the calculations in Part 2 of the appendix, the normal form of (1.2) on the center manifold can be written in polar coordinates as

$$
\dot{\rho}=\left(a_{1} \mu+b_{1} \rho^{2}\right) \rho+O\left(\mu^{2} \rho\right)+O\left(\rho^{4}\right)
$$

where

$$
a_{1}=\frac{\omega_{0} \tau_{0}^{(0)}}{\left(1+\tau_{0}^{(0)}\right)^{2}+\omega_{0}^{2}}>0
$$


Mathematical Problems in Engineering

$$
b_{1}=\frac{f^{\prime \prime \prime}(0)}{2} \frac{\tau_{0}^{(0)}\left(1+\tau_{0}^{(0)}+\omega_{0} \tau_{0}^{(0)}\right)}{\left(1+\tau_{0}^{(0)}\right)^{2}+\omega_{0}^{2}}<0 .
$$

Conclusion (1) then follows immediately.

(2) Let $\tau^{*}=\tau_{0}^{(j)}, u^{*}=\omega_{j} / \tau_{0}^{(j)}, \mu=\tau-\tau_{0}^{(j)}$. We obtain from the calculations in Part 1 of the appendix the normal form of (1.2) on the center manifolds that is given by

$$
\begin{aligned}
\left(\begin{array}{c}
\dot{\omega}_{1} \\
\dot{\omega}_{2}
\end{array}\right)= & u^{*} \tau^{*}\left(\begin{array}{c}
\omega_{2} \\
-\omega_{1}
\end{array}\right)+\mu u^{*}\left(\begin{array}{c}
\operatorname{Im}\left(a^{-1}\right) \omega_{1}+\operatorname{Re}\left(a^{-1}\right) \omega_{2} \\
-\operatorname{Re}\left(a^{-1}\right) \omega_{1}+\operatorname{Im}\left(a^{-1}\right) \omega_{2}
\end{array}\right) \\
& +3 b \tau^{*}\left(\rho_{1}^{2}+2 \rho_{2}^{2}\right)\left(\begin{array}{l}
\operatorname{Re}\left(a^{-1}\left(1-i u^{*}\right)\right) \omega_{1}-\operatorname{Im}\left(a^{-1}\left(1-i u^{*}\right)\right) \omega_{2} \\
\operatorname{Im}\left(a^{-1}\left(1-i u^{*}\right)\right) \omega_{1}+\operatorname{Re}\left(a^{-1}\left(1-i u^{*}\right)\right) \omega_{2}
\end{array}\right) \\
& +O\left(\mu^{2}|\omega|\right)+O\left(|\omega|^{4}\right), \\
\left(\begin{array}{c}
\dot{\omega}_{3} \\
\dot{\omega}_{4}
\end{array}\right)= & u^{*} \tau^{*}\left(\begin{array}{c}
\omega_{4} \\
-\omega_{3}
\end{array}\right)+\mu u^{*}\left(\begin{array}{c}
\operatorname{Im}\left(a^{-1}\right) \omega_{3}+\operatorname{Re}\left(a^{-1}\right) \omega_{4} \\
-\operatorname{Re}\left(a^{-1}\right) \omega_{3}+\operatorname{Im}\left(a^{-1}\right) \omega_{4}
\end{array}\right) \\
& +3 b \tau^{*}\left(\rho_{2}^{2}+2 \rho_{1}^{2}\right)\left(\begin{array}{c}
\operatorname{Re}\left(a^{-1}\left(1-i u^{*}\right)\right) \omega_{3}-\operatorname{Im}\left(a^{-1}\left(1-i u^{*}\right)\right) \omega_{4} \\
\operatorname{Im}\left(a^{-1}\left(1-i u^{*}\right)\right) \omega_{3}+\operatorname{Re}\left(a^{-1}\left(1-i u^{*}\right)\right) \omega_{4}
\end{array}\right) \\
& +O\left(\mu^{2}|\omega|\right)+O\left(|\omega|^{4}\right),
\end{aligned}
$$

where

$$
\begin{aligned}
\rho_{1} & =\sqrt{\omega_{1}^{2}+\omega_{2}^{2}}, \\
\rho_{2} & =\sqrt{\omega_{3}^{2}+\omega_{4}^{2}}, \\
a & =1+\tau^{*}-i u^{*} \tau^{*}, \\
b & =\frac{1}{3 !} f^{\prime \prime \prime}(0) .
\end{aligned}
$$

Introducing the periodic-scaling parameter $\omega$ and letting

$$
\begin{aligned}
& z_{1}(t)=\omega_{1}(s)+i \omega_{2}(s), \\
& z_{2}(t)=\omega_{3}(s)+i \omega_{4}(s)
\end{aligned}
$$

with

$$
s=\left[(1+\omega) u^{*} \tau^{*}\right]^{-1} t
$$


and using a computation process similar to that of Wu et al. [24], we obtain the normal form

$$
\begin{aligned}
& (1+\omega) \dot{z}_{1}=-i z_{1}-i \mu\left(\tau^{*}\right)^{-1} a^{-1} z_{1}+3 b\left(u^{*}\right)^{-1} a^{-1}\left(1-i u^{*}\right)\left(\left|z_{1}\right|^{2}+2\left|z_{2}\right|^{2}\right) z_{1} \\
& (1+\omega) \dot{z}_{2}=-i z_{2}-i \mu\left(\tau^{*}\right)^{-1} a^{-1} z_{2}+3 b\left(u^{*}\right)^{-1} a^{-1}\left(1-i u^{*}\right)\left(\left|z_{2}\right|^{2}+2\left|z_{1}\right|^{2}\right) z_{2} .
\end{aligned}
$$

Let $g: C \oplus C \oplus R \rightarrow C \oplus C$ be given so that $-g\left(z_{1}, z_{2}, \mu\right)$ is the right-hand side of (3.50). Then (3.50) can be written as

$$
(1+\omega) \dot{z}+g(z, \mu)=0
$$

Note that

$$
D_{z} g(0,0)\left(z_{1}, z_{2}\right)=i\left(z_{1}, z_{2}\right), \quad z=\left(z_{1}, z_{2}\right) \in C \oplus C \text {. }
$$

Also note that $g(\cdot, \mu): C \oplus C \rightarrow C \oplus C$ is $D_{n} \times S^{1}$-equivariant with respect to the following $D_{n} \times S^{1}$-action on $C \oplus C$ :

$$
\begin{gathered}
\rho\left(z_{1}, z_{2}\right)=\left(e^{i(2 k \pi / n)} z_{1}, e^{-i(2 k \pi / n)} z_{2}\right), \quad Z_{n}=\langle\rho\rangle \leq D_{n}, \\
\kappa\left(z_{1}, z_{2}\right)=\left(z_{2}, z_{1}\right), \quad Z_{2}=\langle\kappa\rangle \leq D_{n}, \\
e^{i \theta}\left(z_{1}, z_{2}\right)=\left(e^{i \theta} z_{1}, e^{i \theta} z_{2}\right), \quad e^{i \theta} \in S^{1} .
\end{gathered}
$$

Using a computation process similar to that of Wu et al. [24], we obtain

$$
\begin{gathered}
A_{0}=i u^{*}\left(\tau^{*}\right)^{-1} a^{-1}-i \omega, \\
A_{N}=-6 b\left(u^{*}\right)^{-1} a^{-1}\left(1-i u^{*}\right), \\
B_{0}=3 b\left(u^{*}\right)^{-1} a^{-1}\left(1-i u^{*}\right) .
\end{gathered}
$$

By the results of [1, page 376], we know that the bifurcation of phase-locked oscillation is supercritical (resp., subcritical) and depends on whether $\operatorname{Re}\left(A_{N}+B_{0}\right)>0$ (resp., $\operatorname{Re}\left(A_{N}+\right.$ $\left.B_{0}\right)<0$ ), and these are orbitally asymptotically stable if $\operatorname{Re}\left(A_{N}+B_{0}\right)>0$ and $\operatorname{Re} B_{0}<0$. 
Note that

$$
\begin{aligned}
\operatorname{Re}\left(A_{N}+B_{0}\right) & =\operatorname{Re}\left(-3 b\left(u^{*}\right)^{-1} a^{-1}\left(1-i u^{*}\right)\right) \\
& =-3 b\left(u^{*}\right)^{-1} \operatorname{Re}\left[\frac{1+\tau^{*}+i u^{*} \tau^{*}}{\left(1+\tau^{*}\right)^{2}+\left(u^{*} \tau^{*}\right)^{2}}\left(1-i u^{*}\right)\right] \\
& =-3 b\left(u^{*}\right)^{-1} \frac{1+\tau^{*}+\left(u^{*}\right)^{2} \tau^{*}}{\left(1+\tau^{*}\right)^{2}+\left(u^{*} \tau^{*}\right)^{2}} \\
& >0, \\
\operatorname{Re}\left(B_{0}\right) & =\operatorname{Re}\left(3 b\left(u^{*}\right)^{-1} a^{-1}\left(1-i u^{*}\right)\right) \\
& =3 b\left(u^{*}\right)^{-1} \frac{1+\tau^{*}+\left(u^{*}\right)^{2} \tau^{*}}{\left(1+\tau^{*}\right)^{2}+\left(u^{*} \tau^{*}\right)^{2}} \\
& <0 .
\end{aligned}
$$

Consequently, the bifurcation of phase-locked oscillations is supercritical and orbitally asymptotically stable.

Note also that

$$
\begin{aligned}
\operatorname{Re}\left(2 A_{N}+B_{0}\right) & =\operatorname{Re}\left(-9 b\left(u^{*}\right)^{-1} a^{-1}\left(1-i u^{*}\right)\right) \\
& =-9 b\left(u^{*}\right)^{-1} \frac{1+\tau^{*}+\left(u^{*}\right)^{2} \tau^{*}}{\left(1+\tau^{*}\right)^{2}+\left(u^{*} \tau^{*}\right)^{2}} \\
& >0 \\
\operatorname{Re}\left(B_{0}\right) & <0 .
\end{aligned}
$$

We infer from the results of [1, page 376] again that the bifurcations of mirror-reflecting waves and standing waves are supercritical and unstable. This completes the proof.

Using a proof process similar to that of Yuan and Campbell [25], by using Liapunov's second method we can obtain the followings.

Theorem 3.11. If $|\alpha|+2|\beta|<1$, then the trivial solution of (1.2) is global asymptotically stable.

\section{Computer Simulations}

To demonstrate the properties of the Hopf bifurcation in Section 3, we carry out some numerical simulations for a particular case of (1.2) as in following form:

$$
\dot{x}_{i}(t)=-x_{i}(t)+\alpha\left[\tanh \left(x_{i}(t-\tau)\right)\right]+\beta\left[\tanh \left(x_{i-1}(t-\tau)\right)+\tanh \left(x_{i+1}(t-\tau)\right)\right], \quad i(\bmod 3) .
$$




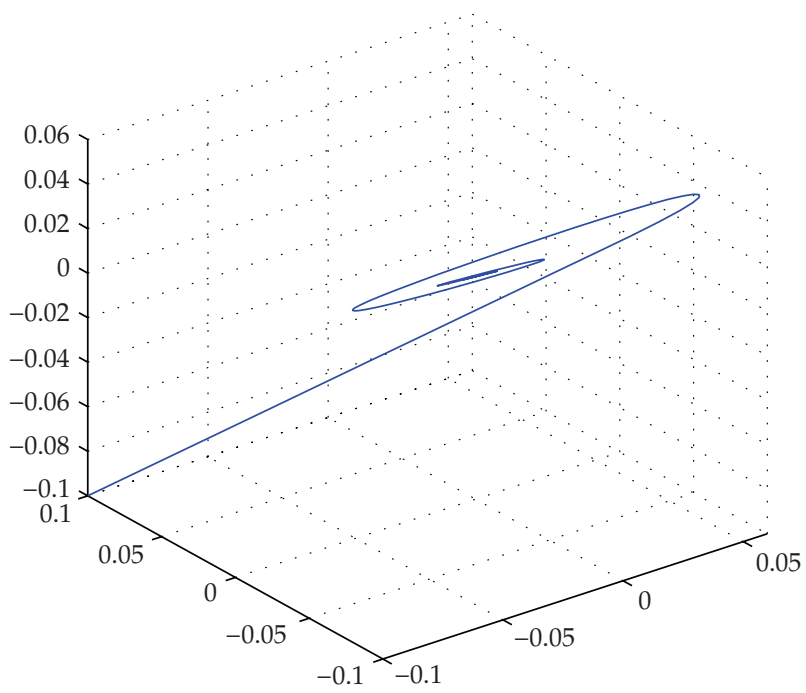

Figure 2: Simulations for the asymptotically stable equilibrium to system (4.1) with $\tau=0.5<\tau_{0}=0.955185$.

Clearly, the origin $(0,0,0)$ is a fixed point to (4.1). Choosing $\alpha=-2$ and $\beta=1 / 3$ we obtain that $\tau_{0}^{(0)}=2.73273$ and $\tau_{0}^{(1)}=0.955185$, thus $\tau_{0}=0.955185$.

From Theorem 3.7(iii), it follows that the zero equilibrium is asymptotically stable if $\tau \in\left[0, \tau_{0}\right)$, it is unstable if $\tau>\tau_{0}$, and the system undergoes a Hopf bifurcation with $\tau=$

$\tau_{k}^{(j)}(j=0,1, \ldots,(n-1) / 2 ; k=0,1, \ldots)$. Simulate the solutions of system (4.1) for $\tau=0.5$ and 1.0. In Figure 2, it is shown that the zero equilibrium is asymptotically stable for $\tau=0.5<$ $\tau_{0}=0.955185$. In Figure 3, for the data $\tau=1.0>\tau_{0}=0.99185$, it is shown that there exists a periodic orbit which is orbitally asymptotically stable.

\section{Appendix}

\section{A. The Calculation of Normal Forms on Center Manifolds with $n$ being an Odd Number}

In this appendix, we employ the algorithm and notations of Faria and Magalhães [26] to derive the normal forms of system (1.2) on the center manifolds.

We first rescale the time by $t \mapsto(t / \tau)$ to normalize the delay so that (1.2) can be written as

$$
\dot{x}(t)=F\left(x_{t}, \tau\right)
$$

in the phase space $C=C\left([-1,0] ; R^{n}\right)$, where for $\phi=\left(\phi_{1}, \phi_{2}, \ldots, \phi_{n}\right)^{T} \in C$, we have

$$
(F(\phi, \tau))_{i}=-\tau \phi_{i}(0)+\alpha \tau f\left(\phi_{i}(-1)\right)+\beta \tau\left[f\left(\phi_{i-1}(-1)\right)+f\left(\phi_{i+1}(-1)\right)\right]
$$




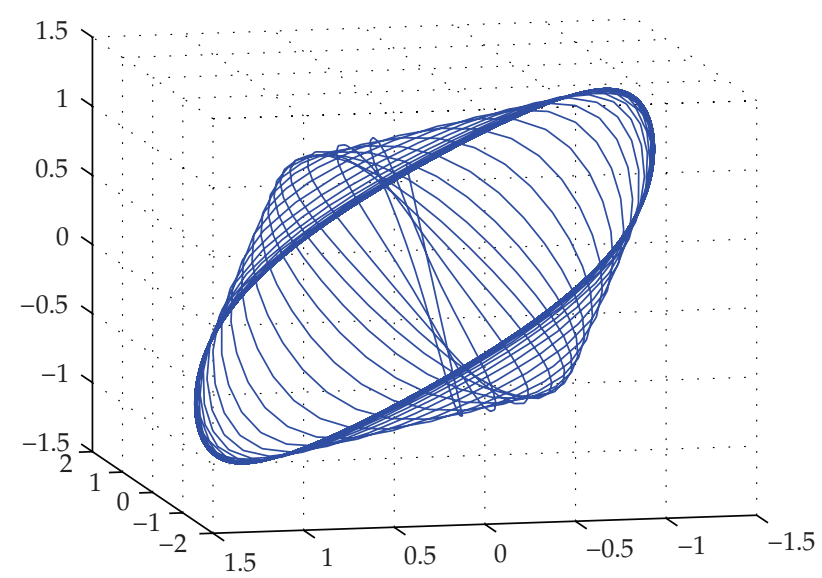

Figure 3: Simulations for an orbitally asymptotically stable periodic orbit to system (4.1) with $\tau=1.0>$ $\tau_{0}=0.99185$.

with $i(\bmod n)$. We also assume that

$$
f(x)=x+b x^{3}+\text { h.o.t. }
$$

with

$$
b=\frac{1}{3 !} f^{\prime \prime \prime}(0)
$$

The linearized equation at zero for system (A.1) is

$$
\dot{x}(t)=L(\tau) x_{t}
$$

where

$$
\begin{aligned}
L(\tau)(\phi) & =-\tau \phi(0)+\tau \alpha \phi(-1)+\tau \beta \delta(\phi(-1)), \\
\delta & =\left(\begin{array}{ccccccc}
0 & 1 & 0 & 0 & \cdots & 0 & 1 \\
1 & 0 & 1 & 0 & \cdots & 0 & 0 \\
0 & 1 & 0 & 1 & \cdots & 0 & 0 \\
0 & 0 & 1 & 0 & \cdots & 0 & 0 \\
0 & 0 & 0 & 1 & \cdots & 0 & 0 \\
\vdots & \vdots & \vdots & \vdots & \cdots & \vdots & \vdots \\
1 & 0 & 0 & 0 & \cdots & 1 & 0
\end{array}\right)_{n \times n} .
\end{aligned}
$$


The characteristic equation of (A.5) at $(0,0, \ldots, 0)^{T}$ is $\operatorname{det} M_{n}(0, \lambda / \tau)=0$, that is,

$$
\left[\frac{\lambda}{\tau}+1-(\alpha+2 \beta) e^{-\lambda}\right] \prod_{j=1}^{(n-1) / 2}\left[\frac{\lambda}{\tau}+1-\left(\alpha+2 \beta \cos \frac{2 \pi j}{n}\right) e^{-\lambda}\right]^{2}=0,
$$

where $\operatorname{det} M_{n}(0, \lambda)=0$ is the characteristic equation of the linearization of (1.2) at $(0,0, \ldots, 0)^{T}$.

\section{Part 1.}

Case $1\left((\alpha, \beta) \in E_{j}(1 \leq j \leq(n-1) / 2)\right.$. In this case, at $\tau=\tau^{*}$ the characteristic equation of (A.5) has imaginary zeros $\pm i u^{*} \tau^{*}$ which are double, where $\tau^{*}=\tau_{0}^{(j)}$ and

$$
u^{*} \tau^{*}=\omega_{j}=\sqrt{\left(\alpha+2 \beta \cos \frac{2 \pi j}{n}\right)^{2}-1}
$$

Since

$$
M_{n}\left(0, i u^{*}\right) v_{j}=\left[i u^{*}+1-\left(\alpha+2 \beta \cos \frac{2 \pi j}{n}\right) e^{-i u^{*} \tau^{*}}\right] v_{j}=0
$$

the center space at $\tau=\tau^{*}$ and in complex coordinates is $X=\operatorname{span}\left(\phi_{1}, \phi_{2}, \phi_{3}, \phi_{4}\right)$, where

$$
\begin{gathered}
\phi_{1}(\theta)=e^{i u^{*} \tau^{*} \theta} v_{j}, \quad \theta \in[-1,0], \\
\phi_{2}(\theta)=e^{-i u^{*} \tau^{*} \theta} \bar{v}_{j}, \quad \theta \in[-1,0], \\
\phi_{3}(\theta)=e^{i u^{*} \tau^{*} \theta} \bar{v}_{j}, \quad \theta \in[-1,0], \\
\phi_{4}(\theta)=e^{-i u^{*} \tau^{*} \theta} v_{j}, \quad \theta \in[-1,0], \\
v_{j}=\left(1, e^{(2 \pi i / n) j}, e^{(2 \pi i / n) 2 j}, \ldots, e^{(2 \pi i / n)(n-1) j}\right)^{T}, \quad j=1,2, \ldots, n-1 .
\end{gathered}
$$

Let

$$
\Phi=\left(\phi_{1}, \phi_{2}, \phi_{3}, \phi_{4}\right)
$$

Note that

$$
\begin{aligned}
\bar{v}_{i}^{T} v_{j}=\left\{\begin{array}{ll}
n, & i=j, \\
0, & i \neq j,
\end{array} \quad i, j=1,2, \ldots, n-1 .\right. \\
\bar{v}_{j}=v_{n-j} .
\end{aligned}
$$


It is easy to check that a basis for the adjoint space $X^{*}$ is

$$
\Psi(s)=\left(\begin{array}{c}
\psi_{1}(s) \\
\psi_{2}(s) \\
\psi_{3}(s) \\
\psi_{4}(s)
\end{array}\right)=\frac{1}{n}\left(\begin{array}{c}
(\bar{a})^{-1} e^{-i u^{*} \tau^{*} s} \bar{v}_{j}^{T} \\
a^{-1} e^{i u^{*} \tau^{*} s} v_{j}^{T} \\
(\bar{a})^{-1} e^{-i u^{*} \tau^{*} s} v_{j}^{T} \\
a^{-1} e^{i u^{*} \tau^{*} s} \bar{v}_{j}^{T}
\end{array}\right), \quad s \in[0,1]
$$

with $(\Psi, \Phi)=\operatorname{Id}$ (the $4 \times 4$ identity matrix) for the adjoint bilinear from on $C^{*} \times C$ defind in [27], where

$$
a=1+\tau^{*}-i u^{*} \tau^{*}
$$

It is useful to note the following:

$$
\Psi(0)=\frac{1}{n}\left(\begin{array}{c}
(\bar{a})^{-1} \bar{v}_{j}^{T} \\
a^{-1} v_{j}^{T} \\
(\bar{a})^{-1} v_{j}^{T} \\
a^{-1} \bar{v}_{j}^{T}
\end{array}\right)
$$

and for $x \in C^{4}$, we have

$$
\begin{gathered}
\Phi(0) x=\left[v_{j}, \bar{v}_{j}, \bar{v}_{j}, v_{j}\right] x=\left(x_{1}+x_{4}\right) v_{j}+\left(x_{2}+x_{3}\right) \bar{v}_{j}, \\
\Phi(-1) x=\left(e^{-i u^{*} \tau^{*}} x_{1}+e^{i u^{*} \tau^{*}} x_{4}\right) v_{j}+\left(e^{i u^{*} \tau^{*}} x_{2}+e^{-i u^{*} \tau^{*}} x_{3}\right) \bar{v}_{j}, \\
\delta(\Phi(-1) x)=\left(e^{-i u^{*} \tau^{*}} x_{1}+e^{i u^{*} \tau^{*}} x_{4}\right) \delta v_{j}+\left(e^{i u^{*} \tau^{*}} x_{2}+e^{-i u^{*} \tau^{*}} x_{3}\right) \delta \bar{v}_{j}=2 \cos \frac{2 \pi j}{n} \Phi(-1) x .
\end{gathered}
$$

Introducing the new parameter

$$
\mu=\tau-\tau^{*},
$$

we can rewrite (A.1) as

$$
\dot{z}(t)=L\left(\tau^{*}\right) z_{t}+G\left(z_{t}, \mu\right),
$$


where

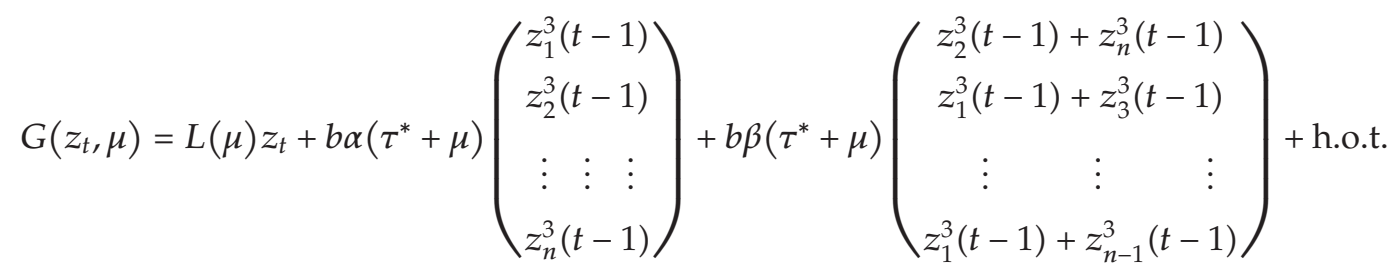

Define the $4 \times 4$ matrix

$$
B=i u^{*} \tau^{*} \operatorname{diag}(1,-1,1,-1) \text {. }
$$

Using the decomposition $z_{t}=\Phi x(t)+y_{t}$, we can decompose (A.18) as

$$
\begin{gathered}
\dot{x}=B x+\Psi(0) G(\Phi x+y, \mu), \\
\dot{y}=A_{Q^{1}} y+(I-\pi) X_{0} G(\Phi x+y, \mu)
\end{gathered}
$$

with $x \in C^{4}, y \in Q^{1}$. Here and throughout this appendix, we refer the readers to [26] for explanations of several notations involved. We will write the Taylor expansion

$$
\Psi(0) G(\Phi x+y, \mu)=\sum_{j \geq 2} \frac{1}{j !} f_{j}^{1}(x, y, \mu)
$$

where $f_{j}^{1}(x, y, \mu)$ are homogeneous polynomials of degree $j$ in $(x, y, \mu)$ with coefficients in $C^{4}$. Then the normal form of (1.2) on the center manifold of the origin at $\mu=0$ is given by

$$
\dot{x}=B x+\frac{1}{2} g_{2}^{1}(x, 0, \mu)+\frac{1}{3 !} g_{3}^{1}(x, 0, \mu)+\text { h.o.t., }
$$

where $g_{2}^{1}$ and $g_{3}^{1}$ will be calculated in the following part of this section. 
First of all, using $\delta(\Phi(-1) x)=2 \cos (2 \pi j / n) \Phi(-1) x$, we find that

$$
\begin{aligned}
\frac{1}{2} f_{2}^{1}(x, 0, \mu)= & \Psi(0) L(\mu)(\Phi x) \\
= & \Psi(0) \mu[-\Phi(0) x+\alpha \Phi(-1) x+\beta \delta(\Phi(-1) x)] \\
= & \Psi(0) \mu\left\{\left[-\left(x_{1}+x_{4}\right) v_{j}-\left(x_{2}+x_{3}\right) \bar{v}_{j}\right]+\left(\alpha+2 \beta \cos \frac{2 \pi j}{n}\right)\right. \\
& \left.\times\left[\left(e^{-i u^{*} \tau^{*}} x_{1}+e^{i u^{*} \tau^{*}} x_{4}\right) v_{j}+\left(e^{i u^{*} \tau^{*}} x_{2}+e^{-i u^{*} \tau^{*}} x_{3}\right) \bar{v}_{j}\right]\right\} \\
= & \mu i u^{*} \Psi(0)\left[\left(x_{1}-x_{4}\right) v_{j}+\left(-x_{2}+x_{3}\right) \bar{v}_{j}\right] \\
= & i \mu u^{*}\left(\begin{array}{c}
(\bar{a})^{-1}\left(x_{1}-x_{4}\right) \\
a^{-1}\left(-x_{2}+x_{3}\right) \\
(\bar{a})^{-1}\left(-x_{2}+x_{3}\right) \\
a^{-1}\left(x_{1}-x_{4}\right)
\end{array}\right) .
\end{aligned}
$$

These are the second-order terms in $(\mu, x)$ of (A.22), and following Faria and Magalhaes [26], we have the second-order terms in $(\mu, x)$ of the normal form on center manifold as follows:

$$
\frac{1}{2} g_{2}^{1}(x, 0, \mu)=\operatorname{Proj}_{\operatorname{ker}\left(M_{2}^{1}\right)} \frac{1}{2} f_{2}^{1}(x, 0, \mu) .
$$

Here we recall that

$$
M_{j}^{1}(p)(x, \mu)=D_{x} p(x, \mu) B x-B p(x, \mu), \quad j \geq 2 .
$$

In particular,

$$
M_{j}^{1}\left(\mu x^{q} e_{k}\right)=i u^{*} \tau^{*} \mu\left(q_{1}-q_{2}+q_{3}-q_{4}+(-1)^{k}\right) x^{q} e_{k}, \quad|q|=j-1
$$

where $j \geq 2,1 \leq k \leq 4$, and $\left\{e_{1}, e_{2}, e_{3}, e_{4}\right\}$ are the canonical basis for $C^{4}$. Therefore, if $|q|=1$, then

$$
\begin{gathered}
\operatorname{ker}\left(M_{2}^{1}\right) \cap \operatorname{span}\left\{\mu x^{q} e_{k}:|q|=1, k=1,2,3,4\right\} \\
=\operatorname{span}\left\{\mu x_{1} e_{1}, \mu x_{3} e_{1}, \mu x_{2} e_{2}, \mu x_{4} e_{2}, \mu x_{1} e_{3}, \mu x_{3} e_{3}, \mu x_{2} e_{4}, \mu x_{4} e_{4}\right\} \\
\frac{1}{2} g_{2}^{1}(x, 0, \mu)=i \mu u^{*}\left(\begin{array}{c}
(\bar{a})^{-1} x_{1} \\
-a^{-1} x_{2} \\
(\bar{a})^{-1} x_{3} \\
-a^{-1} x_{4}
\end{array}\right) .
\end{gathered}
$$


To compute $g_{3}^{1}(x, 0, \mu)$, we first note that from (A.26) it follows that

$$
\begin{aligned}
g_{3}^{1}(x, 0, \mu) & =\operatorname{Proj}_{\operatorname{ker}\left(M_{3}^{1}\right)} \overline{f_{3}^{1}}(x, 0, \mu) \\
& =\operatorname{Proj}_{\operatorname{ker}\left(M_{3}^{1}\right)} \overline{f_{3}^{1}}(x, 0,0)+O\left(\mu^{2}|x|\right),
\end{aligned}
$$

since $\mu x^{q} e_{j}$ is not in $\operatorname{Ker}\left(M_{3}^{1}\right)$, for $|q|=2, j=1,2,3,4$. Next, we define

$$
\overline{f_{3}^{1}}(x, 0, \mu)=f_{3}^{1}(x, 0, \mu)+\frac{3}{2}\left[\left(D_{x} f_{2}^{1}\right) U_{2}^{1}-\left(D_{x} U_{2}^{1}\right) g_{2}^{1}\right](x, 0, \mu)+\frac{3}{2}\left[\left(D_{y} f_{2}^{1}\right) h\right](x, 0, \mu),
$$

where $U_{2}^{1}$ is the change of variables associated with the transformation from $f_{2}^{1}$ to $g_{2}^{1}$, and $h$ is such that $M_{2}^{2}(h)=g_{2}^{2}$, that is, $h=U_{2}^{2}$ is the change of variables associated with the transformation of the second-order terms in the second equation of system (A.21). For $\mu=0$, $f_{2}^{1}(x, 0,0)=g_{2}^{1}(x, 0,0)=0$, and we have simply

$$
\frac{1}{3 !} f_{3}^{1}(x, 0,0)=\frac{1}{3 !} f_{3}^{1}(x, 0,0)=b \tau^{*} \Psi(0)\left[\beta \delta\left((\Phi(-1) x)^{3}\right)+\alpha(\Phi(-1) x)^{3}\right]
$$

where we utilized the following notations:

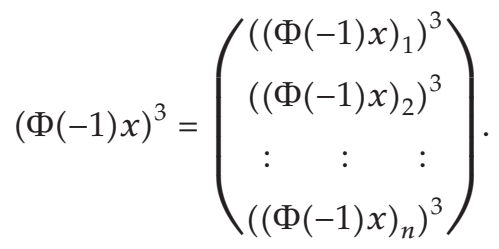

Let

$$
A_{1}=e^{-i u^{*} \tau^{*}} x_{1}+e^{i u^{*} \tau^{*}} x_{4}, \quad A_{2}=e^{i u^{*} \tau^{*}} x_{2}+e^{-i u^{*} \tau^{*}} x_{3} .
$$


We have

$$
\begin{aligned}
(\Phi(-1) x)^{3}= & \left(\begin{array}{c}
\left(A_{1}+A_{2}\right)^{3} \\
\left(e^{(2 \pi i / n) j} A_{1}+e^{-(2 \pi i / n) j} A_{2}\right)^{3} \\
\left(e^{(2 \pi i / n) 2 j} A_{1}+e^{-(2 \pi i / n) 2 j} A_{2}\right)^{3} \\
\vdots
\end{array} \begin{array}{c}
\vdots \\
\vdots \\
\left(e^{(2 \pi i / n)(n-1) j} A_{1}+e^{-(2 \pi i / n)(n-1) j} A_{2}\right)^{3}
\end{array}\right) \\
& =\left(\begin{array}{c}
A_{1}^{3} v_{j}^{3}+3 A_{1}^{2} A_{2} v_{j}+A_{2}^{3} \bar{v}_{j}^{3}+3 A_{1} A_{2}^{2} \bar{v}_{j}, \\
\left(e^{(2 \pi i / n) j} A_{1}+e^{-(2 \pi i / n) j} A_{2}\right)^{3}+\left(e^{(2 \pi i / n)(n-1) j} A_{1}+e^{-(2 \pi i / n)(n-1) j} A_{2}\right)^{3} \\
\left(A_{1}+A_{2}\right)^{3}+\left(e^{(2 \pi i / n) 2 j} A_{1}+e^{-(2 \pi i / n) 2 j} A_{2}\right)^{3} \\
\left(e^{(2 \pi i / n) j} A_{1}+e^{-(2 \pi i / n) j} A_{2}\right)^{3}+\left(e^{(2 \pi i / n) 3 j} A_{1}+e^{-(2 \pi i / n) 3 j} A_{2}\right)^{3} \\
\vdots \\
\vdots \\
\left(A_{1}+A_{2}\right)^{3}+\left(e^{(2 \pi i / n)(n-2) j} A_{1}+e^{-(2 \pi i / n)(n-2) j} A_{2}\right)^{3}
\end{array}\right.
\end{aligned}
$$

Then, we have

$$
\begin{aligned}
\Psi(0)(\Phi(-1) x)^{3} & =\frac{1}{n}\left(\begin{array}{c}
(\bar{a})^{-1} \bar{v}_{j}^{T} \\
a^{-1} v_{j}^{T} \\
(\bar{a})^{-1} v_{j}^{T} \\
a^{-1} \bar{v}_{j}^{T}
\end{array}\right)\left(A_{1}^{3} v_{j}^{3}+3 A_{1}^{2} A_{2} v_{j}+A_{2}^{3} \bar{v}_{j}^{3}+3 A_{1} A_{2}^{2} \bar{v}_{j}\right) \\
& =3\left(\begin{array}{c}
(\bar{a})^{-1} A_{1}^{2} A_{2} \\
a^{-1} A_{1} A_{2}^{2} \\
(\bar{a})^{-1} A_{1} A_{2}^{2} \\
a^{-1} A_{1}^{2} A_{2}
\end{array}\right),
\end{aligned}
$$

$$
\begin{aligned}
\Psi(0) \delta(\Phi(-1) x)^{3} & =\left[(\Psi(0) \delta)^{T}\right]^{T}(\Phi(-1) x)^{3} \\
& =\left[\delta(\Psi(0))^{T}\right]^{T}(\Phi(-1) x)^{3}
\end{aligned}
$$




$$
\begin{aligned}
& =\left[\frac{1}{n} \delta\left((\bar{a})^{-1} \bar{v}_{j}, a^{-1} v_{j},(\bar{a})^{-1} v_{j}, a^{-1} \bar{v}_{j}\right)\right]^{T}(\Phi(-1) x)^{3} \\
& =\left[\frac{1}{n} 2 \cos \frac{2 \pi j}{n}\left((\bar{a})^{-1} \bar{v}_{j}, a^{-1} v_{j},(\bar{a})^{-1} v_{j}, a^{-1} \bar{v}_{j}\right)\right]^{T}(\Phi(-1) x)^{3} \\
& =\frac{1}{n} 2 \cos \frac{2 \pi j}{n}\left(\begin{array}{c}
(\bar{a})^{-1} \bar{v}_{j}^{T} \\
a^{-1} v_{j}^{T} \\
(\bar{a})^{-1} v_{j}^{T} \\
a^{-1} \bar{v}_{j}^{T}
\end{array}\right)\left(A_{1}^{3} v_{j}^{3}+3 A_{1}^{2} A_{2} v_{j}+A_{2}^{3} \bar{v}_{j}^{3}+3 A_{1} A_{2}^{2} \bar{v}_{j}\right) \\
& =6 \cos \frac{2 \pi j}{n}\left(\begin{array}{c}
(\bar{a})^{-1} A_{1}^{2} A_{2} \\
a^{-1} A_{1} A_{2}^{2} \\
(\bar{a})^{-1} A_{1} A_{2}^{2} \\
a^{-1} A_{1}^{2} A_{2}
\end{array}\right) .
\end{aligned}
$$

Then, we have

$$
\begin{aligned}
\frac{1}{3 !} f_{3}^{1}(x, 0,0) & =b \tau^{*} \Psi(0)\left[\beta \delta\left((\Phi(-1) x)^{3}\right)+\alpha(\Phi(-1) x)^{3}\right] \\
& =b \tau^{*} 3\left(\alpha+2 \beta \cos \frac{2 \pi j}{n}\right)\left(\begin{array}{c}
(\bar{a})^{-1} A_{1}^{2} A_{2} \\
a^{-1} A_{1} A_{2}^{2} \\
(\bar{a})^{-1} A_{1} A_{2}^{2} \\
a^{-1} A_{1}^{2} A_{2}
\end{array}\right) .
\end{aligned}
$$

Note that

$$
\begin{aligned}
& A_{1}^{2} A_{2}=\left(e^{-2 i u^{*} \tau^{*}} x_{1}^{2}+2 x_{1} x_{4}+e^{2 i u^{*} \tau^{*}} x_{4}^{2}\right)\left(e^{i u^{*} \tau^{*}} x_{2}+e^{-i u^{*} \tau^{*}} x_{3}\right), \\
& A_{1} A_{2}^{2}=\left(e^{-i u^{*} \tau^{*}} x_{1}+e^{i u^{*} \tau^{*}} x_{4}\right)\left(e^{2 i u^{*} \tau^{*}} x_{2}^{2}+2 x_{2} x_{3}+e^{-2 i u^{*} \tau^{*}} x_{3}^{2}\right)
\end{aligned}
$$

Also note that

$$
M_{3}^{1}\left(x^{q} e_{j}\right)=0, \quad \text { with }|q|=3 \text { iff } q_{1}-q_{2}+q_{3}-q_{4}+(-1)^{j}=0, j=1,2,3,4
$$


Then

$$
\begin{aligned}
\frac{1}{3 !} g_{3}^{1}(x, 0,0) & =\operatorname{Proj}_{\operatorname{ker}\left(M_{2}^{1}\right)} \frac{1}{3 !} f_{3}^{1}(x, 0,0) \\
& =3 b \tau^{*}\left(\alpha+2 \beta \cos \frac{2 \pi j}{n}\right)\left(\begin{array}{c}
(\bar{a})^{-1} e^{-i u^{*} \tau^{*}}\left(x_{1}^{2} x_{2}+2 x_{1} x_{3} x_{4}\right) \\
a^{-1} e^{i u^{*} \tau^{*}}\left(x_{1} x_{2}^{2}+2 x_{2} x_{3} x_{4}\right) \\
(\bar{a})^{-1} e^{-i u^{*} \tau^{*}}\left(x_{3}^{2} x_{4}+2 x_{1} x_{2} x_{3}\right) \\
a^{-1} e^{i u^{*} \tau^{*}}\left(x_{3} x_{4}^{2}+2 x_{1} x_{2} x_{4}\right)
\end{array}\right) \\
& =3 b \tau^{*}\left(\begin{array}{c}
(\bar{a})^{-1}\left(1+i u^{*}\right) x_{1}\left(x_{1} x_{2}+2 x_{3} x_{4}\right) \\
a^{-1}\left(1-i u^{*}\right) x_{2}\left(x_{1} x_{2}+2 x_{3} x_{4}\right) \\
(\bar{a})^{-1}\left(1+i u^{*}\right) x_{3}\left(x_{3} x_{4}+2 x_{1} x_{2}\right) \\
a^{-1}\left(1-i u^{*}\right) x_{4}\left(x_{3} x_{4}+2 x_{1} x_{2}\right)
\end{array}\right) .
\end{aligned}
$$

Consequently, the normal form on the center manifold becomes

$$
\begin{aligned}
\dot{x}= & i u^{*} \tau^{*}\left(\begin{array}{rrrr}
1 & 0 & 0 & 0 \\
0 & -1 & 0 & 0 \\
0 & 0 & 1 & 0 \\
0 & 0 & 0 & -1
\end{array}\right) x+i \mu u^{*}\left(\begin{array}{l}
(\bar{a})^{-1} x_{1} \\
-a^{-1} x_{2} \\
(\bar{a})^{-1} x_{3} \\
-a^{-1} x_{4}
\end{array}\right) \\
& +\frac{1}{3 !} g_{3}^{1}(x, 0,0)+O\left(\mu^{2}|x|\right)+O\left(|x|^{4}\right)
\end{aligned}
$$

for $x \in C^{4}$. Changing to real coordinates by the change of variables

$$
x=S \omega, \quad \text { with } S=\left(\begin{array}{rrrc}
1 & -i & 0 & 0 \\
1 & i & 0 & 0 \\
0 & 0 & 1 & -i \\
0 & 0 & 1 & i
\end{array}\right)
$$

and letting

$$
\begin{aligned}
& \rho_{1}^{2}=x_{1} x_{2}=\omega_{1}^{2}+\omega_{2}^{2}, \\
& \rho_{2}^{2}=x_{3} x_{4}=\omega_{3}^{2}+\omega_{4}^{2},
\end{aligned}
$$


we obtain

$$
\begin{aligned}
\left(\begin{array}{c}
\dot{\omega}_{1} \\
\dot{\omega}_{2}
\end{array}\right)= & u^{*} \tau^{*}\left(\begin{array}{c}
\omega_{2} \\
-\omega_{1}
\end{array}\right)+\mu u^{*}\left(\begin{array}{c}
\operatorname{Im}\left(a^{-1}\right) \omega_{1}+\operatorname{Re}\left(a^{-1}\right) \omega_{2} \\
-\operatorname{Re}\left(a^{-1}\right) \omega_{1}+\operatorname{Im}\left(a^{-1}\right) \omega_{2}
\end{array}\right) \\
& +3 b \tau^{*}\left(\rho_{1}^{2}+2 \rho_{2}^{2}\right)\left(\begin{array}{c}
\operatorname{Re}\left(a^{-1}\left(1-i u^{*}\right)\right) \omega_{1}-\operatorname{Im}\left(a^{-1}\left(1-i u^{*}\right)\right) \omega_{2} \\
\operatorname{Im}\left(a^{-1}\left(1-i u^{*}\right)\right) \omega_{1}+\operatorname{Re}\left(a^{-1}\left(1-i u^{*}\right)\right) \omega_{2}
\end{array}\right) \\
& +O\left(\mu^{2}|\omega|\right)+O\left(|\omega|^{4}\right), \\
\left(\begin{array}{c}
\dot{\omega}_{3} \\
\dot{\omega}_{4}
\end{array}\right)= & u^{*} \tau^{*}\left(\begin{array}{c}
\omega_{4} \\
-\omega_{3}
\end{array}\right)+\mu u^{*}\left(\begin{array}{c}
\operatorname{Im}\left(a^{-1}\right) \omega_{3}+\operatorname{Re}\left(a^{-1}\right) \omega_{4} \\
-\operatorname{Re}\left(a^{-1}\right) \omega_{3}+\operatorname{Im}\left(a^{-1}\right) \omega_{4}
\end{array}\right) \\
& +3 b \tau^{*}\left(\rho_{2}^{2}+2 \rho_{1}^{2}\right)\left(\begin{array}{c}
\operatorname{Re}\left(a^{-1}\left(1-i u^{*}\right)\right) \omega_{3}-\operatorname{Im}\left(a^{-1}\left(1-i u^{*}\right)\right) \omega_{4} \\
\operatorname{Im}\left(a^{-1}\left(1-i u^{*}\right)\right) \omega_{3}+\operatorname{Re}\left(a^{-1}\left(1-i u^{*}\right)\right) \omega_{4}
\end{array}\right) \\
& +O\left(\mu^{2}|\omega|\right)+O\left(|\omega|^{4}\right) .
\end{aligned}
$$

If we use double polar coordinates

$$
\begin{aligned}
& \omega_{1}=\rho_{1} \cos \chi_{1}, \\
& \omega_{2}=\rho_{1} \sin \chi_{1}, \\
& \omega_{3}=\rho_{2} \cos \chi_{2}, \\
& \omega_{4}=\rho_{2} \sin \chi_{2},
\end{aligned}
$$

then we find

$$
\begin{gathered}
\dot{\rho}_{1}=\left(a_{1} \mu+b_{1} \rho_{1}^{2}+2 b_{1} \rho_{2}^{2}\right) \rho_{1}+O\left(\mu^{2}\left|\left(\rho_{1}, \rho_{2}\right)\right|\right)+O\left(\left|\left(\rho_{1}, \rho_{2}\right)\right|^{4}\right), \\
\dot{\rho}_{2}=\left(a_{1} \mu+b_{1} \rho_{2}^{2}+2 b_{1} \rho_{1}^{2}\right) \rho_{2}+O\left(\mu^{2}\left|\left(\rho_{1}, \rho_{2}\right)\right|\right)+O\left(\left|\left(\rho_{1}, \rho_{2}\right)\right|^{4}\right), \\
\dot{X}_{1}=-u^{*} \tau^{*}+c_{1} \mu+d_{1} \rho_{1}^{2}+2 d_{1} \rho_{2}^{2}+O\left(\mu^{2}\left|\left(\rho_{1}, \rho_{2}\right)\right|\right)+O\left(\left|\left(\rho_{1}, \rho_{2}\right)\right|^{4}\right), \\
\dot{X}_{2}=-u^{*} \tau^{*}+c_{1} \mu+d_{1} \rho_{2}^{2}+2 d_{1} \rho_{1}^{2}+O\left(\mu^{2}\left|\left(\rho_{1}, \rho_{2}\right)\right|\right)+O\left(\left|\left(\rho_{1}, \rho_{2}\right)\right|^{4}\right),
\end{gathered}
$$

with

$$
\begin{gathered}
a_{1}=\operatorname{Im}\left(a^{-1}\right) u^{*}, \quad b_{1}=\operatorname{Re}\left(a^{-1}\left(1-i u^{*}\right)\right) 3 b \tau^{*}, \\
c_{1}=-u^{*} \operatorname{Re}\left(a^{-1}\right), \quad d_{1}=3 b \tau^{*} \operatorname{Im}\left(a^{-1}\left(1-i u^{*}\right)\right) .
\end{gathered}
$$


As

$$
a^{-1}=\frac{1+\tau^{*}+i u^{*} \tau^{*}}{\left(1+\tau^{*}\right)^{2}+\left(u^{*} \tau^{*}\right)^{2}}
$$

we have

$$
\begin{gathered}
a_{1}=\frac{\left(u^{*}\right)^{2} \tau^{*}}{\left(1+\tau^{*}\right)^{2}+\left(u^{*} \tau^{*}\right)^{2}} \\
b_{1}=3 b \tau^{*} \frac{1+\tau^{*}+\left(u^{*}\right)^{2} \tau^{*}}{\left(1+\tau^{*}\right)^{2}+\left(u^{*} \tau^{*}\right)^{2}} \\
c_{1}=-\frac{u^{*}\left(1+\tau^{*}\right)}{\left(1+\tau^{*}\right)^{2}+\left(u^{*} \tau^{*}\right)^{2}} \\
d_{1}=-3 b \frac{u^{*} \tau^{*}}{\left(1+\tau^{*}\right)^{2}+\left(u^{*} \tau^{*}\right)^{2}}
\end{gathered}
$$

\section{Part 2.}

Case $2\left((\alpha, \beta) \in E_{0}\right)$. In this case, at $\tau=\tau^{*}$ the characteristic equation of (A.5) has imaginary zeros $\pm i u^{*} \tau^{*}$ which are simple, where $\tau^{*}=\tau_{0}^{(0)}$ and

$$
u^{*} \tau^{*}=\omega_{0}=\sqrt{(\alpha+2 \beta)^{2}-1}
$$

are given by (3.5) and (3.7), respectively. Since

$$
M_{n}\left(0, i u^{*}\right) v_{0}=\left[i u^{*}+1-(\alpha+2 \beta) e^{-i u^{*} \tau^{*}}\right] v_{0}=0
$$

where $v_{0}=(1,1, \ldots, 1)^{T}$, the center space at $\tau=\tau^{*}$ and in complex coordinates is $X=$ $\operatorname{span}\left(\phi_{1}, \phi_{2}\right)$, where

$$
\begin{aligned}
& \phi_{1}(\theta)=e^{i u^{*} \tau^{*} \theta} v_{0}, \\
& \phi_{2}(\theta)=e^{-i u^{*} \tau^{*} \theta} v_{0},
\end{aligned}
$$

Let

$$
\Phi=\left(\phi_{1}, \phi_{2}\right)
$$

From $v_{0}^{T} v_{0}=n$, it is easy to check that a basis for the adjoint space $X^{*}$ is

$$
\Psi(s)=\left(\begin{array}{l}
\psi_{1}(s) \\
\psi_{2}(s)
\end{array}\right)=\frac{1}{n}\left(\begin{array}{c}
(\bar{a})^{-1} e^{-i u^{*} \tau^{*} s} v_{0}^{T} \\
a^{-1} e^{i u^{*} \tau^{*} s} v_{0}^{T}
\end{array}\right), \quad s \in[0,1]
$$


with $(\Psi, \Phi)=\operatorname{Id}$ (the $2 \times 2$ identity matrix) for the adjoint bilinear from on $C^{*} \times C$ defined in [27], where

$$
a=1+\tau^{*}-i u^{*} \tau^{*}
$$

It is useful to note the following:

$$
\Psi(0)=\frac{1}{n}\left(\begin{array}{c}
(\bar{a})^{-1} v_{0}^{T} \\
a^{-1} v_{0}^{T}
\end{array}\right)
$$

and for $x \in C^{2}$, we have

$$
\begin{gathered}
\Phi(0) x=\left[v_{0}, v_{0}\right] x=\left(x_{1}+x_{2}\right) v_{0} \\
\Phi(-1) x=\left(e^{-i u^{*} \tau^{*}} x_{1}+e^{i u^{*} \tau^{*}} x_{2}\right) v_{0} \\
\delta(\Phi(-1)) x=2\left(e^{-i u^{*} \tau^{*}} x_{1}+e^{i u^{*} \tau^{*}} x_{2}\right) v_{0}
\end{gathered}
$$

For the new parameter $\mu=\tau-\tau^{*}$ and decomposition $z_{t}=\Phi x(t)+y_{t}, x \in C^{2}, y \in Q^{1}$, and with

$$
B=\operatorname{diag}\left(i u^{*} \tau^{*},-i u^{*} \tau^{*}\right),
$$

the normal form of (1.2) on the center manifold of the origin at $\mu=0$ is

$$
\dot{x}=B x+\frac{1}{2} g_{2}^{1}(x, 0, \mu)+\frac{1}{3 !} g_{3}^{1}(x, 0, \mu)+\text { h.o.t., }
$$

and we will compute the second- and third-order terms, that is, $g_{2}^{1}(x, 0, \mu)$ and $g_{3}^{1}(x, 0, \mu)$, as we have done above for Case 2 of Theorem 3.10 We have

$$
\begin{aligned}
\frac{1}{2} f_{2}^{1}(x, 0, \mu) & =\Psi(0) L(\mu)(\Phi x) \\
& =\Psi(0) \mu[-\Phi(0) x+\alpha \Phi(-1) x+\beta \delta(\Phi(-1) x)] \\
& =\Psi(0) \mu\left[-\left(x_{1}+x_{2}\right)+(\alpha+2 \beta)\left(e^{-i u^{*} \tau^{*}} x_{1}+e^{i u^{*} \tau^{*}} x_{2}\right)\right] v_{0} \\
& =\Psi(0) \mu\left[-\left(x_{1}+x_{2}\right)+\left(1+i u^{*}\right) x_{1}+\left(1-i u^{*}\right) x_{2}\right] v_{0} \\
& =i \mu u^{*}\left(\begin{array}{c}
(\bar{a})^{-1}\left(x_{1}-x_{2}\right) \\
a^{-1}\left(x_{1}-x_{2}\right)
\end{array}\right) .
\end{aligned}
$$


Since

$$
\begin{gathered}
\frac{1}{2} g_{2}^{1}(x, 0, \mu)=\operatorname{Proj}_{\operatorname{ker}\left(M_{2}^{1}\right)} \frac{1}{2} f_{2}^{1}(x, 0, \mu), \\
M_{j}^{1}\left(\mu x^{q} e_{k}\right)=i u^{*} \tau^{*} \mu\left(q_{1}-q_{2}+(-1)^{k}\right) x^{q} e_{k}, \quad|q|=j-1, k=1,2, j \geq 2
\end{gathered}
$$

for the canonical basis $\left\{e_{1}, e_{2}\right\}$ for $C^{2}$, then

$$
\begin{gathered}
\operatorname{ker}\left(M_{2}^{1}\right) \cap \operatorname{span}\left\{\mu x^{q} e_{k}:|q|=1, k=1,2\right\}=\operatorname{span}\left\{\mu x_{1} e_{1}, \mu x_{2} e_{2}\right\} \\
\frac{1}{2} g_{2}^{1}(x, 0, \mu)=i \mu u^{*}\left(\begin{array}{c}
(\bar{a})^{-1} x_{1} \\
-a^{-1} x_{2}
\end{array}\right) .
\end{gathered}
$$

As for the previous case and for similar reasons,

$$
\begin{aligned}
g_{3}^{1}(x, 0, \mu) & =\operatorname{Proj}_{\operatorname{ker}\left(M_{3}^{1}\right)} \overline{f_{3}^{1}}(x, 0, \mu) \\
& =\operatorname{Proj}_{\operatorname{ker}\left(M_{3}^{1}\right)} f_{3}^{1}(x, 0,0)+O\left(\mu^{2}|x|\right),
\end{aligned}
$$

where

$$
\begin{aligned}
\frac{1}{3 !} f_{3}^{1}(x, 0,0) & =b \tau^{*} \Psi(0)\left[\beta \delta\left((\Phi(-1) x)^{3}\right)+\alpha(\Phi(-1) x)^{3}\right] \\
& =b \tau^{*} \Psi(0)(\alpha+2 \beta)\left(e^{-i u^{*} \tau^{*}} x_{1}+e^{i u^{*} \tau^{*}} x_{2}\right)^{3} v_{0} \\
& =b \tau^{*}(\alpha+2 \beta)\left(e^{-i u^{*} \tau^{*}} x_{1}+e^{i u^{*} \tau^{*}} x_{2}\right)^{3}\left(\begin{array}{c}
(\bar{a})^{-1} \\
a^{-1}
\end{array}\right) .
\end{aligned}
$$

Also note that

$$
M_{3}^{1}\left(x^{q} e_{j}\right)=i u^{*} \tau^{*}\left(q_{1}-q_{2}+(-1)^{j}\right) x^{q} e_{j}, \quad|q|=3, j=1,2,
$$

which implies that

$$
\operatorname{ker}\left(M_{3}^{1}\right) \cap \operatorname{span}\left\{x^{q} e_{j}:|q|=3, j=1,2\right\}=\operatorname{span}\left\{x_{1}^{2} x_{2} e_{1}, x_{1} x_{2}^{2} e_{2}\right\}
$$

We can then derive

$$
\frac{1}{3 !} g_{3}^{1}(x, 0,0)=3 b \tau^{*}\left(\begin{array}{c}
(\bar{a})^{-1}\left(1+i u^{*}\right) x_{1}^{2} x_{2} \\
a^{-1}\left(1-i u^{*}\right) x_{1} x_{2}^{2}
\end{array}\right)
$$


Consequently, the normal form on the center manifold becomes

$$
\begin{aligned}
\dot{x}= & i u^{*} \tau^{*}\left(\begin{array}{cc}
1 & 0 \\
0 & -1
\end{array}\right) x+i \mu u^{*}\left(\begin{array}{c}
(\bar{a})^{-1} x_{1} \\
-a^{-1} x_{2}
\end{array}\right) \\
& +\frac{1}{3 !} g_{3}^{1}(x, 0,0)+O\left(\mu^{2}|x|\right)+O\left(|x|^{4}\right)
\end{aligned}
$$

for $x \in C^{2}$. Changing to real coordinates by the change of variables

$$
x=S \omega, \quad \text { with } S=\left(\begin{array}{cc}
1 & -i \\
1 & i
\end{array}\right),
$$

and letting

$$
\rho^{2}=x_{1} x_{2}=\omega_{1}^{2}+\omega_{2}^{2}
$$

we obtain

$$
\begin{aligned}
\left(\begin{array}{c}
\dot{\omega}_{1} \\
\dot{\omega}_{2}
\end{array}\right)= & u^{*} \tau^{*}\left(\begin{array}{c}
\omega_{2} \\
-\omega_{1}
\end{array}\right)+\mu u^{*}\left(\begin{array}{c}
\operatorname{Im}\left(a^{-1}\right) \omega_{1}+\operatorname{Re}\left(a^{-1}\right) \omega_{2} \\
-\operatorname{Re}\left(a^{-1}\right) \omega_{1}+\operatorname{Im}\left(a^{-1}\right) \omega_{2}
\end{array}\right) \\
& +3 b \tau^{*} \rho^{2}\left(\begin{array}{l}
\operatorname{Re}\left(a^{-1}\left(1-i u^{*}\right)\right) \omega_{1}-\operatorname{Im}\left(a^{-1}\left(1-i u^{*}\right)\right) \omega_{2} \\
\operatorname{Im}\left(a^{-1}\left(1-i u^{*}\right)\right) \omega_{1}+\operatorname{Re}\left(a^{-1}\left(1-i u^{*}\right)\right) \omega_{2}
\end{array}\right) \\
& +O\left(\mu^{2}|\omega|\right)+O\left(|\omega|^{4}\right)
\end{aligned}
$$

If we use polar coordinates

$$
\begin{aligned}
& \omega_{1}=\rho \cos x, \\
& \omega_{2}=\rho \sin x,
\end{aligned}
$$

then we find that

$$
\begin{gathered}
\dot{\rho}=\left(a_{1} \mu+b_{1} \rho^{2}\right) \rho+O\left(\mu^{2} \rho\right)+O\left(\rho^{4}\right), \\
\dot{X}=-u^{*} \tau^{*}+c_{1} \mu+d_{1} \rho^{2}+O\left(\mu^{2} \rho\right)+O\left(\rho^{4}\right),
\end{gathered}
$$

where $a_{1}, b_{1}, c_{1}$, and $d_{1}$ are as in (A.48). 


\section{Acknowledgments}

This research was supported by the National Natural Science Foundations of China (no. 10771045), the Program of Excellent Team in Harbin Institute of Technology, and the Harbin Institute Technology (Weihai) Science Foundation (no. ZB200812).

\section{References}

[1] M. Golubitsky, I. Stewart, and D. G. Schaeffer, Singularities and Groups in Bifurcation Theory. Vol. II, vol. 69 of Applied Mathematical Sciences, Springer, New York, NY, USA, 1988.

[2] W. Krawcewicz, P. Vivi, and J. Wu, "Computation formulae of an equivariant degree with applications to symmetric bifurcations," Nonlinear Studies, vol. 4, no. 1, pp. 89-119, 1997.

[3] W. Krawcewicz and J. Wu, "Theory and applications of Hopf bifurcations in symmetric functionaldifferential equations," Nonlinear Analysis: Theory, Methods E Applications, vol. 35, no. 7, pp. 845-870, 1999.

[4] J. Wu, "Symmetric functional-differential equations and neural networks with memory," Transactions of the American Mathematical Society, vol. 350, no. 12, pp. 4799-4838, 1998.

[5] M. A. Cohen and S. Grossberg, "Absolute stability of global pattern formation and parallel memory storage by competitive neural networks," IEEE Transactions on Systems, Man, and Cybernetics, vol. 13, no. 5, pp. 815-826, 1983.

[6] S. Grossberg, "Competition, decision, and consensus," Journal of Mathematical Analysis and Applications, vol. 66, no. 2, pp. 470-493, 1978.

[7] S. Grossberg, "Biological competition: decision rules, pattern formation, and oscillations," Proceedings of the National Academy of Sciences of the United States of America, vol. 77, no. 4, pp. 2338-2342, 1980.

[8] J. J. Hopfield, "Neurons with graded response have collective computational properties like those of two-state neurons," Proceedings of the National Academy of Sciences of the United States of America, vol. 81, no. 10, pp. 3088-3092, 1984.

[9] J. J. Hopfield, "Neural networks and physical systems with emergent collective computational abilities," Proceedings of the National Academy of Sciences of the United States of America, vol. 79, no. 8, pp. 2554-2558, 1982.

[10] S. A. Campbell, S. Ruan, and J. Wei, "Qualitative analysis of a neural network model with multiple time delays," International Journal of Bifurcation and Chaos in Applied Sciences and Engineering, vol. 9, no. 8, pp. 1585-1595, 1999.

[11] Y. Chen and J. Wu, "Existence and attraction of a phase-locked oscillation in a delayed network of two neurons," Differential and Integral Equations, vol. 14, no. 10, pp. 1181-1236, 2001.

[12] Y. Chen and J. Wu, "Minimal instability and unstable set of a phase-locked periodic orbit in a delayed neural network," Physica D, vol. 134, no. 2, pp. 185-199, 1999.

[13] Y. Chen and J. Wu, "Slowly oscillating periodic solutions for a delayed frustrated network of two neurons," Journal of Mathematical Analysis and Applications, vol. 259, no. 1, pp. 188-208, 2001.

[14] D. Fan and J. Wei, "Hopf bifurcation analysis in a tri-neuron network with time delay," Nonlinear Analysis: Real World Applications, vol. 9, no. 1, pp. 9-25, 2008.

[15] T. Faria, "On a planar system modelling a neuron network with memory," Journal of Differential Equations, vol. 168, no. 1, pp. 129-149, 2000.

[16] S. Guo and L. Huang, "Hopf bifurcating periodic orbits in a ring of neurons with delays," Physica D, vol. 183, no. 1-2, pp. 19-44, 2003.

[17] S. Guo and L. Huang, "Stability of nonlinear waves in a ring of neurons with delays," Journal of Differential Equations, vol. 236, no. 2, pp. 343-374, 2007.

[18] X. Li and J. Wei, "Stability and bifurcation analysis on a delayed neural network model," International Journal of Bifurcation and Chaos in Applied Sciences and Engineering, vol. 15, no. 9, pp. 2883-2893, 2005.

[19] S. Ruan and J. Wei, "On the zeros of transcendental functions with applications to stability of delay differential equations with two delays," Dynamics of Continuous, Discrete E Impulsive Systems. Series A, vol. 10, no. 6, pp. 863-874, 2003.

[20] J. Wei and M. Y. Li, "Global existence of periodic solutions in a tri-neuron network model with delays," Physica D, vol. 198, no. 1-2, pp. 106-119, 2004.

[21] J. Wei and S. Ruan, "Stability and bifurcation in a neural network model with two delays," Physica D, vol. 130, no. 3-4, pp. 255-272, 1999. 
[22] J. Wei and C. Zhang, "Bifurcation analysis of a class of neural networks with delays," Nonlinear Analysis: Real World Applications, vol. 9, no. 5, pp. 2234-2252, 2008.

[23] Y. Yuan and J. Wei, "Multiple bifurcation analysis in a neural network model with delays," International Journal of Bifurcation and Chaos in Applied Sciences and Engineering, vol. 16, no. 10, pp. 2903-2913, 2006.

[24] J. Wu, T. Faria, and Y. S. Huang, "Synchronization and stable phase-locking in a network of neurons with memory," Mathematical and Computer Modelling, vol. 30, no. 1-2, pp. 117-138, 1999.

[25] Y. Yuan and S. A. Campbell, "Stability and synchronization of a ring of identical cells with delayed coupling," Journal of Dynamics and Differential Equations, vol. 16, no. 3, pp. 709-744, 2004.

[26] T. Faria and L. T. Magalhães, "Normal forms for retarded functional-differential equations with parameters and applications to Hopf bifurcation," Journal of Differential Equations, vol. 122, no. 2, pp. 181-200, 1995.

[27] J. Hale and S. M. Verduyn Lunel, Introduction to Functional Differential Equations, Springer, New York, NY, USA, 1993. 


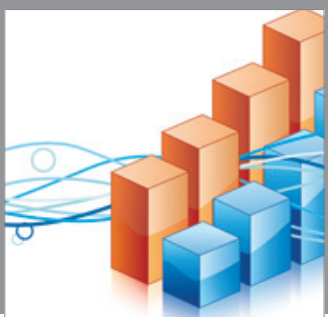

Advances in

Operations Research

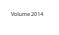

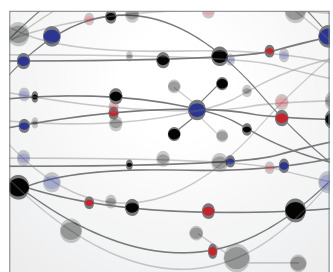

\section{The Scientific} World Journal
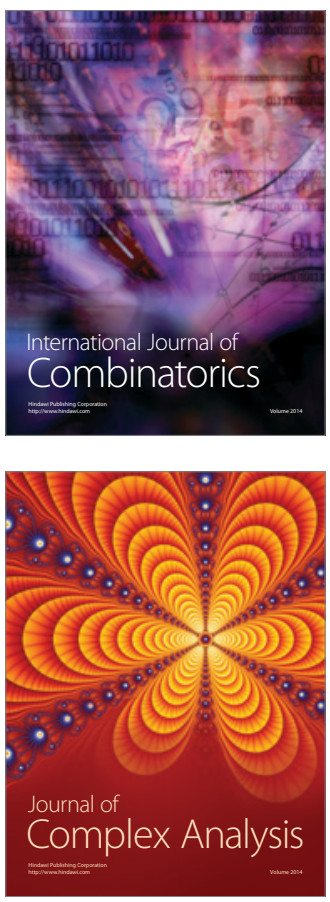

International Journal of

Mathematics and

Mathematical

Sciences
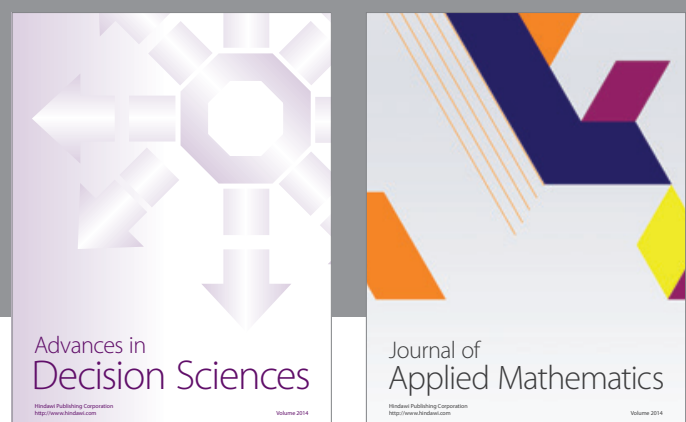

Journal of

Applied Mathematics
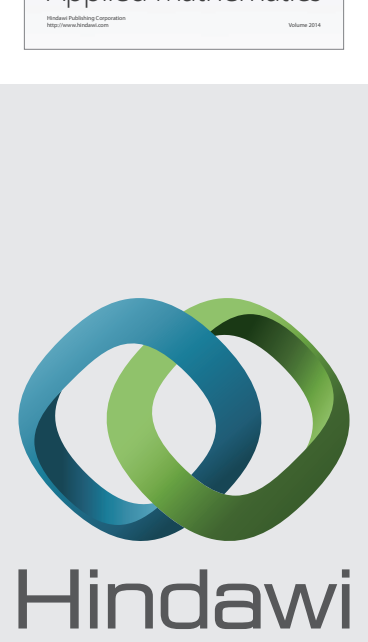

Submit your manuscripts at http://www.hindawi.com
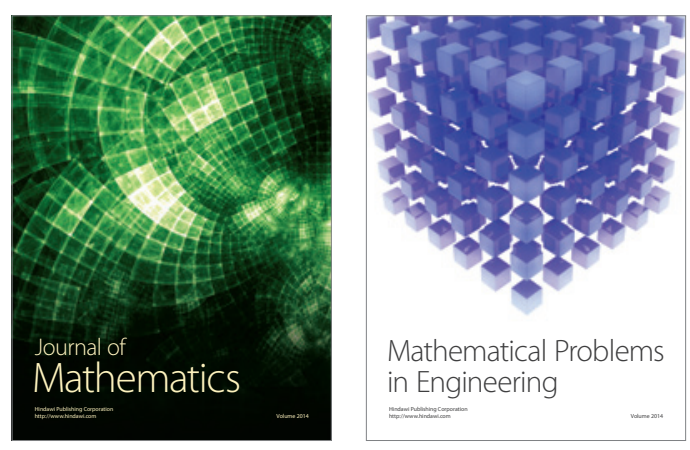

Mathematical Problems in Engineering
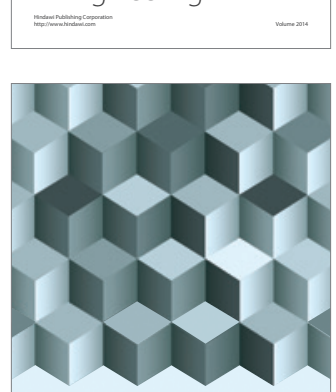

Journal of

Function Spaces
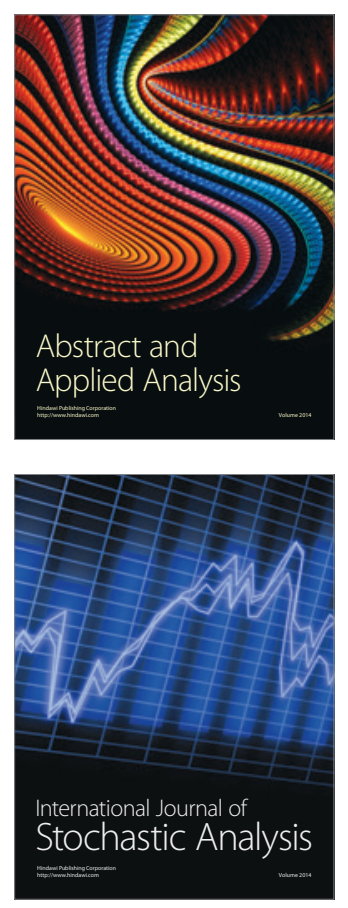

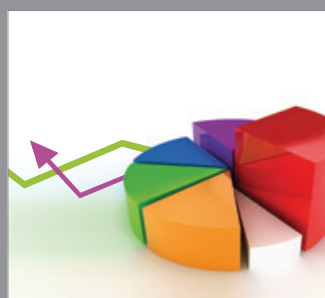

ournal of

Probability and Statistics

Promensencen
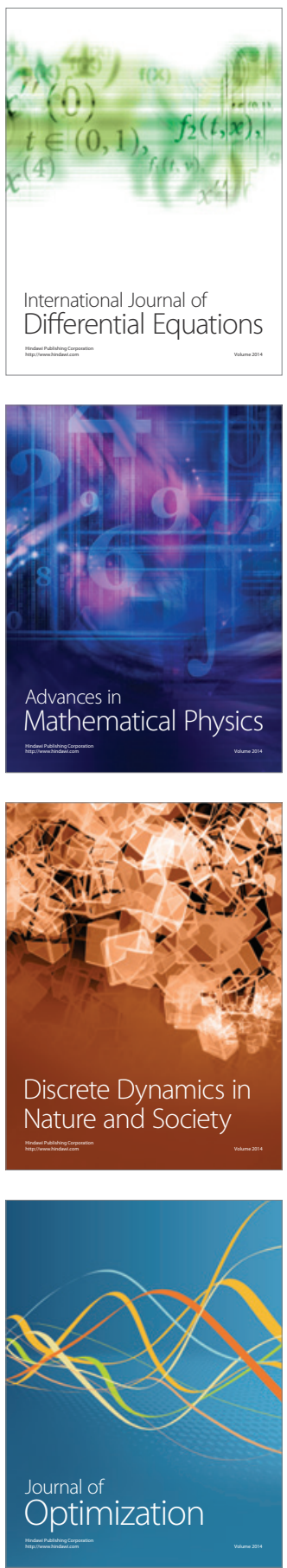